CNS Spectrums (2017), 22, 348-362. C Cambridge University Press 2016. The online version of this article is published within an Open Access environment subject to the conditions of the Creative Commons Attribution-NonCommercial-ShareAlike licence <http://creativecommons.org/licenses/by-nc-sa/3.0/>. The written permission of Cambridge University Press must be obtained for commercial re-use. doi:10.1017/S1092852916000626

\title{
Efficacy, safety, and tolerability of vortioxetine for the treatment of major depressive disorder in patients aged 55 years or older
}

\author{
George G. Nomikos, ${ }^{\text {* Dapo Tomori, }}{ }^{2}$ Wei Zhong, ${ }^{3}$ John Affinito, ${ }^{4}$ \\ and William Palo ${ }^{5}$
}

\footnotetext{
${ }^{1}$ Clinical Science, Takeda Development Center Americas, Deerfield, Illinois, USA

${ }^{2}$ U.S. Medical Affairs, Takeda Development Center Americas, Deerfield, Illinois, USA

${ }^{3}$ Statistics, Takeda Development Center Americas, Deerfield, Illinois, USA

${ }^{4}$ Pharmacovigilance, Takeda Development Center Americas, Deerfield, Illinois, USA

${ }^{5}$ Safety Statistics, Takeda Development Center Americas, Deerfield, Illinois, USA
}

Objective. These post hoc analyses evaluate the efficacy, safety, and tolerability of vortioxetine versus placebo in patients aged $\geq 55$ years with major depressive disorder (MDD).

Methods. Study-level efficacy data from 12 short-term, fixed-dose, randomized, placebo-controlled trials of vortioxetine 5-20 mg/day were assessed using a random-effects meta-analysis. Adverse events (AEs), vital signs, ECG values, liver enzymes, and body weight were pooled from the same studies. Patients had baseline Montgomery-Åsberg Depression Rating Scale (MADRS) total scores ranging from 22-30.

Results. 1508 patients (mean age $=62.4$ years; range, $55-88$ years) were included. Mean differences from placebo in change from baseline to study end (6/8 weeks) in MADRS were $-2.56(5 \mathrm{mg}, \mathrm{n}=324, P=0.035),-2.87(10 \mathrm{mg}$, $\mathrm{n}=222, P=0.007),-1.32(15 \mathrm{mg}, \mathrm{n}=90, P=\mathrm{NS})$, and $-4.65(20 \mathrm{mg}, \mathrm{n}=165, P=0.012)$. Odds ratios for response versus placebo were $1.6(5 \mathrm{mg}, P=\mathrm{NS}), 1.8(10 \mathrm{mg}, P=0.002), 1.2(15 \mathrm{mg}, P=\mathrm{NS})$, and $2.5(20 \mathrm{mg}, P<0.001)$, and for remission versus placebo were $1.5(5 \mathrm{mg}, P=\mathrm{NS}), 1.5(10 \mathrm{mg}, P=\mathrm{NS}), 1.4(15 \mathrm{mg}, P=\mathrm{NS})$, and $2.7(20 \mathrm{mg}$, $P=0.001$ ). The proportion of patients with AEs for placebo and vortioxetine $5-20 \mathrm{mg}$ was $61.5 \%$ and $62.3 \%$, respectively, with no increase at increased doses. Vortioxetine demonstrated a placebo-level incidence of serious AEs (1.2\%). AEs occurring in $\geq 5 \%$ of any treatment group were nausea, headache, diarrhea, dizziness, dry mouth, constipation, fatigue, vomiting, and anxiety. No clinically significant mean changes in vital signs, ECG values, liver enzymes, or body weight emerged during treatment.

Conclusion. Vortioxetine $5-20 \mathrm{mg}$ /day is efficacious and well tolerated in MDD patients aged $\geq 55$ years, a group that is often comorbid with other conditions and treated with other medications.

Received 27 June 2016; Accepted 3 August 2016; First published online 21 November 2016

Key words: Late-life depression, major depressive disorder, meta-analysis, multimodal antidepressant, older adults, vortioxetine.

\footnotetext{
* Address for correspondence: George G. Nomikos, MD, PhD, Takeda Development Center Americas, One Takeda Parkway, Deerfield, IL 60015, USA.

(Email: gnomikos1@gmail.com)

Assistance with manuscript preparation was provided by Ann C. Sherwood, PhD, Nicole Coolbaugh, and Philip Sjostedt, BPharm, with The Medicine Group and was paid for by the Takeda Pharmaceutical Company, Ltd. and H. Lundbeck A/S.

This study was funded by the Takeda Pharmaceutical Company, Ltd. and H. Lundbeck A/S.
}

\section{Introduction}

Major depressive disorder (MDD) in older adults is a growing public health concern as the global population ages. The United Nations estimates that $16.9 \%$ of the world's population was aged 55 or older in $2015 .{ }^{1}$ By 2050 , this is projected to exceed $27 \%$ of the global population. ${ }^{1}$ Global estimates suggest that MDD affects almost $7 \%$ of the individuals aged 60 years or older worldwide and 
accounts for $5.7 \%$ of years lived with disability. ${ }^{2}$ Trends are similar in the United States; in 2012, $14 \%$ of the US population was over 65 and $26 \%$ was over 55 years of age. ${ }^{3}$ By 2030 , more than $20 \%$ of Americans are expected to be over 65 years old. ${ }^{4}$ Two analyses of nationally representative samples recently found that Americans aged 55 years or older had 12-month prevalence of MDD between $4.0 \%$ and $5.6 \% .^{5,6}$ When MDD prevalence was stratified by 10 -year age intervals within this older US population, the highest prevalence was found in the subgroup aged 55 to 64 years $(6.2 \%$ and $7.4 \%)$, and declined in older age groups. ${ }^{5,6}$ The 12-month prevalence was also found to be higher for women than for men. 5

Diagnosis of MDD in older patients carries a high risk of comorbid psychiatric and nonpsychiatric illnesses. These patients also report lower quality of life ${ }^{7}$ and higher health care expenditures than non-depressed elderly. ${ }^{8,9}$ In the US, patients aged 55 years or older with MDD were 4 times more likely to experience another mood disorder and more than twice as likely to experience an anxiety disorder during 3 years of study follow-up when compared with individuals with no depression. ${ }^{10}$ They were also more than 3.5 times as likely to attempt suicide during follow-up. ${ }^{10} \mathrm{MDD}$ in older individuals is associated with substantial disability related to cognitive dysfunction ${ }^{7,11-13}$ and functional impairment. $^{14,15}$

Sinnige $e t a l^{16}$ reported that $82 \%$ of patients aged 55 years or older diagnosed with MDD were also diagnosed with at least 2 other chronic diseases, with an average of 3 co-occurring diseases (including MDD). The likelihood of having depression increases with the number of chronic conditions, regardless of age. ${ }^{16,17} \mathrm{~A}$ recent analysis of electronic health records from patients seen in a primary care practice found that the contribution of chronic diseases to the risk of MDD differed somewhat by age. Individuals aged 46-60 years with MDD were significantly more likely to have ischemic heart disease (odds ratio [OR], 2.4; 95\% confidence interval [CI], 1.7-3.5), diabetes mellitus (OR 2.1; 95\% CI, 1.6-2.8), asthma (OR 1.7; 95\% CI, 1.2-2.4), and rheumatoid arthritis/osteoarthritis (OR 1.5; 95\% CI, 1.2-1.9) than age-matched controls. Among patients with MDD who were aged $>60$ years, there was an increased risk of stroke (OR 1.9; 95\% CI, 1.4-2.7), heart failure (OR 2.2; 95\% CI, 1.6-3.0), and rheumatoid arthritis/osteoarthritis (OR 1.5; 95\% CI, 1.3-1.8). ${ }^{18}$ In addition, all-cause mortality rates are higher in older patients with depression than those without, independent of comorbid illnesses. In a longitudinal cohort analysis, after adjustment for cardiovascular disease and diabetes, the risk of mortality in individuals with depression was found to be 1.75 times higher than in individuals without depression during a 2-year follow-up interval. ${ }^{19}$
Data to guide antidepressant prescribing in patients aged older than 55 are limited, with ongoing clinical research being conducted to identify potential predictors of clinical response in this patient population. ${ }^{20-22}$ Selective serotonin reuptake inhibitors (SSRIs) and serotoninnorepinephrine reuptake inhibitors (SNRIs) are generally considered safe and well tolerated in older patients. However, potential side effects are not entirely benign. SSRIs, particularly citalopram, have been associated with cardiac arrhythmias, especially at higher doses. ${ }^{23-25}$ A 10-year study showed a dose-dependent association between use of SSRIs or SNRIs and fragility fracture (hazard ratio, 1.88; 95\% CI, 1.48-2.39) after adjusting for a higher propensity for falls and other risk factors in this population. ${ }^{26}$ In addition, SSRIs and SNRIs are recommended to be used with caution in older patients with diminished kidney function to avoid hyponatremia. ${ }^{27}$ It is, however, unknown whether these warnings should be considered class-related for patients older than 55, or precautions for consideration when managing these patients.

Two meta-analyses ${ }^{28,29}$ assessed the efficacy of antidepressants in late-life depression, defined as aged $\geq 55$ years and $\geq 60$ years, respectively. Results from both suggested that antidepressants are effective in older patients, but that efficacy diminishes with age. Vortioxetine safety and efficacy have been evaluated in an 8-week, short-term, randomized, double-blind, placebocontrolled study (NCT00811252) conducted specifically in patients aged $\geq 65$ years with moderate-to-severe MDD (mean baseline Montgomery-Åsberg Depression Rating Scale [MADRS] total score $\sim 30) .{ }^{30}$ In that trial $(\mathrm{N}=453)$, vortioxetine significantly improved depression symptoms (treatment difference versus placebo in change from baseline in Hamilton Depression Rating Scale-24 item $\left[\right.$ HAM-D ${ }_{24}$ ] total score, $-3.3 ; P<0.0011$ ) at Week 8. Vortioxetine was safe and well tolerated in this age group.

Vortioxetine was approved in 2013 in the US for the treatment of adults with MDD and in the European Union for the treatment of a major depressive episode (MDE) in adults. The mechanism of action of vortioxetine is related to its multimodal activity, which combines 2 pharmacological actions: direct modulation of receptor activity and inhibition of the serotonin (5-HT) transporter. In addition to inhibiting the 5 -HT transporter, vortioxetine is an antagonist at $5-\mathrm{HT}_{3}, 5-\mathrm{HT}_{7}$, and $5-\mathrm{HT}_{1 \mathrm{D}}$ receptors; a partial agonist at $5-\mathrm{HT}_{1 \mathrm{~B}}$ receptors; and an agonist at $5-\mathrm{HT}_{1 \mathrm{~A}}$ receptors. ${ }^{31-33}$

To evaluate vortioxetine in a larger population of patients with late-life depression, these post hoc analyses used data from 12 short-term, randomized, double-blind, placebo-controlled, fixed-dose studies in MDD of up to 8 weeks' duration, including study NCT00811252..$^{30,34-44}$ These analyses assessed vortioxetine $5-20 \mathrm{mg} /$ day in the population of individuals aged 55 years or older. 


\section{Methods}

Twelve randomized, short-term (6 or 8 weeks), fixeddose, placebo-controlled trials were included in this meta-analysis, and the study designs are summarized in Table 1, including treatment period and dosing information. Details of the individual studies have been published in peer-reviewed journals. ${ }^{30,34-44}$ All studies were designed, conducted, and reported in accordance with the Declaration of Helsinki ${ }^{45}$ and in compliance with the International Conference on Harmonisation guidelines for Good Clinical Practice. ${ }^{46}$

The 12 studies utilized for this meta-analysis used similar inclusion and exclusion criteria: patients had to be aged 1875 years (inclusive, except NCT00811252 recruited patients aged 65 years or older and NCT01255787 recruited those aged 20-64 years) and meet the Diagnostic and Statistical Manual of Mental Disorders, Fourth Edition, Text Revision (DSM-IV-TR) criteria for an MDE lasting at least 4 weeks (NCT00811252) or 3 months (all other studies). Patients were required to have a MADRS ${ }^{47}$ total score $\geq 22 \quad$ (NCT00672620), $\geq 30 \quad$ (NCT00839423 and NCT00672958), or $\geq 26$ (all other studies). Five studies also required a Clinical Global Impressions-Severity of Illness $(\text { CGI-S })^{48}$ score of $\geq 4$ (NCT01140906, NCT01153009, NCT01163266, NCT01179516, and NCT01255787). While 5 of the studies (NCT00635219, NCT01140906, NCT01153009, NCT00672620, and NCT00811252) in this meta-analysis did include duloxetine as an active reference for validation purposes, no direct comparison was made between the comparative safety and efficacy profiles of vortioxetine and duloxetine, as none of the clinical trials were specifically designed to directly compare the two agents. As such, no direct comparative claims can be made between vortioxetine and duloxetine.

Patients in these studies were excluded if they had any current psychiatric disorder other than MDD as defined in the DSM-IV-TR; a current or past history of a manic or hypomanic episode, schizophrenia, or any other psychiatric disorder, mental retardation, organic mental disorders, or mental disorders due to a general medical condition; any current diagnosis of substance abuse or dependence as defined in DSM-IV-TR; the presence or history of a clinically significant neurological disorder; any neurodegenerative disorder; or any DSM-IV-TR Axis II disorder, including personality disorder, that might compromise their participation in the study. Patients were also excluded if they had a clinically significant unstable illness (eg, hepatic impairment or renal insufficiency, or a cardiovascular, pulmonary, gastrointestinal, endocrine, neurological, rheumatologic, immunologic, infectious, skin, or subcutaneous tissue disorder, or a metabolic disturbance). Patients with chronic, but stable, disease (such as diabetes, hypertension, hypercholesterolemia, or respiratory diseases) were able to be enrolled at the investigators' discretion.
The predefined primary efficacy endpoints at end of study in the individual studies were the MADRS total score, the HAM-D ${ }_{24}{ }^{49}$ total score, or a composite z-score of the Digit Symbol Substitution Test (DSST) ${ }^{50}$ and Rey Auditory Verbal Learning Test (RAVLT) ${ }^{51}$ scores, with the MADRS as a predefined secondary endpoint in those trials that utilized the HAM-D ${ }_{24}$ or DSST/RAVLT. Only patients randomized to vortioxetine $5-20 \mathrm{mg} /$ day (approved therapeutic dosages) were included in these analyses.

\section{Population characteristics}

Details of baseline demographics, concurrent medical conditions, and concomitant medications were included to describe the type of patient population included in these analyses.

\section{Efficacy outcomes}

For this post hoc meta-analysis, the primary efficacy outcome was defined as the difference from placebo in change from baseline on MADRS total score at study endpoint (week 6 or 8). Secondary efficacy outcomes included difference from placebo in change from baseline on select MADRS individual item scores, MADRS response (defined as $\geq 50 \%$ decrease from baseline in MADRS total score), and MADRS remission (defined as MADRS total score $\leq 10$ ) at study endpoint. We undertook an analysis of select MADRS items, corresponding to symptoms that are related to more severe disability in older MDD patients, as reported by Anderson et al. ${ }^{52}$

\section{Safety and tolerability outcomes}

Safety and tolerability were assessed by the nature and severity of treatment-emergent adverse events (TEAEs) and changes in vital signs, laboratory values, electrocardiograms (ECGs), and body weight. Discontinuations due to TEAEs were also evaluated as a measure of tolerability.

\section{Statistical analysis}

Efficacy was analyzed using the full analysis set (FAS), which includes all randomized patients who took at least 1 dose of study medication and had at least 1 valid postbaseline value of the primary efficacy outcome. The primary statistical methodology in the individual studies was either a mixed effect model for repeated measures (MMRM) or an analysis of covariance (ANCOVA) using last observation carried forward (LOCF); however, all studies performed both methodologies as part of the statistical analysis plan.

This efficacy meta-analysis used aggregated study-level data using MMRM for the individual studies as the primary methodology. The MMRM model used an 
TABLE 1. Summary characteristics of the 12 short-term, fixed-dose, placebo-controlled studies of vortioxetine in patients with MDD included in the meta-analysis (APTS)

\begin{tabular}{|c|c|c|c|c|c|}
\hline NCT identifier & Study duration and mean age ${ }^{a}$ & Dose ${ }^{\mathrm{b}} \mathrm{mg} / \mathrm{day}(\mathrm{N})$ & Inclusion criteria & Primary efficacy endpoint & Reference \\
\hline NCT00839423 & $\begin{array}{l}6 \text { weeks } \\
43.3(11.5)\end{array}$ & $\begin{array}{l}\text { VOR } 5(108) \\
\text { VOR } 10(100) \\
\text { VEN } 225(113) \\
\text { PBO (105) }\end{array}$ & $\begin{array}{l}\text { MADRS } \geq 30 \\
\text { MDE } \geq 3 \text { months and }<12 \text { months } \\
\text { Aged } 18-65 \text { y }\end{array}$ & MADRS & 34 \\
\hline NCT00635219 & $\begin{array}{l}8 \text { weeks } \\
44.9(12.7)\end{array}$ & $\begin{array}{l}\text { VOR } 2.5(155) \\
\text { VOR } 5(157) \\
\text { VOR } 10(151) \\
\text { DUL } 60 \text { (155) } \\
\text { PBO (148) }\end{array}$ & $\begin{array}{l}\text { MADRS } \geq 26 \\
\text { MDE } \geq 3 \text { months } \\
\text { Aged } 18-75 y\end{array}$ & MADRS & 35 \\
\hline NCT00735709 & $\begin{array}{l}8 \text { weeks } \\
46.4(12.1)\end{array}$ & $\begin{array}{l}\text { VOR } 1(140) \\
\text { VOR } 5(140) \\
\text { VOR } 10(139) \\
\text { PBO (140) }\end{array}$ & $\begin{array}{l}\text { MADRS } \geq 26 \\
\text { MDE } \geq 3 \text { months } \\
\text { Aged } 18-75 y\end{array}$ & HAM- $\mathrm{D}_{24}$ & 37 \\
\hline NCT01140906 & $\begin{array}{l}8 \text { weeks } \\
46.7(13.7)\end{array}$ & $\begin{array}{l}\text { VOR } 15(151) \\
\text { VOR } 20(151) \\
\text { DUL } 60(147) \\
\text { PBO (158) }\end{array}$ & $\begin{array}{l}\text { MADRS } \geq 26 \\
\text { CGI-S } \geq 4 \\
\text { MDE }>3 \text { months } \\
\text { recurrent } \\
\text { Aged } 18-75 y\end{array}$ & MADRS & 36 \\
\hline NCT01153009 & $\begin{array}{l}8 \text { weeks } \\
42.9(12.4)\end{array}$ & $\begin{array}{l}\text { VOR } 15(147) \\
\text { VOR } 20(154) \\
\text { DUL } 60(150) \\
\text { PBO (159) }\end{array}$ & $\begin{array}{l}\text { MADRS } \geq 26 \\
\text { CGI-S } \geq 4 \\
\text { MDE } \geq 3 \text { months } \\
\text { recurrent } \\
\text { Aged } 18-75 y\end{array}$ & MADRS & 40 \\
\hline NCT01163266 & $\begin{array}{l}8 \text { weeks } \\
42.8(12.2)\end{array}$ & $\begin{array}{l}\text { VOR } 10(155) \\
\text { VOR } 20(150) \\
\text { PBO (157) }\end{array}$ & $\begin{array}{l}\text { MADRS } \geq 26 \\
\text { CGI }-S \geq 4 \\
\text { MDE } \geq 3 \text { months } \\
\text { recurrent } \\
\text { Aged } 18-75 y\end{array}$ & MADRS & 38 \\
\hline NCT00672958 & $\begin{array}{l}6 \text { weeks } \\
42.4 \text { (12.9) }\end{array}$ & $\begin{array}{l}\text { VOR } 5 \text { (299) } \\
\text { PBO (298) }\end{array}$ & $\begin{array}{l}\text { MADRS } \geq 30 \\
\text { MDE } \geq 3 \text { months } \\
\text { Aged } 18-75 y\end{array}$ & HAM- $\mathrm{D}_{24}$ & 39 \\
\hline NCT00672620 & $\begin{array}{l}8 \text { weeks } \\
42.7(13.7)\end{array}$ & $\begin{array}{l}\text { VOR } 2.5(149) \\
\text { VOR } 5 \text { (153) } \\
\text { DUL } 60(150) \\
\text { PBO (151) }\end{array}$ & $\begin{array}{l}\text { MADRS } \geq 22 \\
\text { MDE } \geq 3 \text { months } \\
\text { Aged } 18-75 y\end{array}$ & HAM- $\mathrm{D}_{24}$ & 41 \\
\hline NCT01179516 & $\begin{array}{l}8 \text { weeks } \\
45.1 \text { (12.4) }\end{array}$ & $\begin{array}{l}\text { VOR } 10(154) \\
\text { VOR } 15(151) \\
\text { PBO (160) }\end{array}$ & $\begin{array}{l}\text { MADRS } \geq 26 \\
\text { CGI-S } \geq 4 \\
\text { MDE } \geq 3 \text { months } \\
\text { recurrent } \\
\text { Aged } 18-75 y\end{array}$ & MADRS & 44 \\
\hline NCT01255787 & $\begin{array}{l}8 \text { weeks } \\
44.4(11.5)\end{array}$ & $\begin{array}{l}\text { VOR } 5(144) \\
\text { VOR } 10(148) \\
\text { VOR } 20(150) \\
\text { PBO (152) }\end{array}$ & $\begin{array}{l}\text { MADRS } \geq 26 \\
\text { CGI-S } \geq 4 \\
\text { MDE } \geq 3 \text { months } \\
\text { Aged } 20-64 y\end{array}$ & MADRS & 43 \\
\hline NCT00811252 & $\begin{array}{l}8 \text { weeks } \\
70.6(4.9)\end{array}$ & $\begin{array}{l}\text { VOR } 5(156) \\
\text { DUL } 60(151) \\
\text { PBO (145) }\end{array}$ & $\begin{array}{l}\text { MADRS } \geq 26 \\
\text { MDE } \geq 4 \text { weeks } \\
\text { recurrent } \\
\text { Aged } \geq 65 \text { y }\end{array}$ & HAM- $\mathrm{D}_{24}$ & 30 \\
\hline NCT01422213 & $\begin{array}{l}8 \text { weeks } \\
45.7(12.0)\end{array}$ & $\begin{array}{l}\text { VOR } 10(195) \\
\text { VOR } 20(207) \\
\text { PBO (196) }\end{array}$ & $\begin{array}{l}\text { MADRS } \geq 26 \\
\text { MDE } \geq 3 \text { months } \\
\text { recurrent } \\
\text { Aged } 18-65 y\end{array}$ & $\begin{array}{l}\text { DSST and RAVLT } \\
\text { composite }\end{array}$ & 42 \\
\hline
\end{tabular}

${ }^{a}$ Mean (standard deviation) age for the total population (all treatment groups combined).

${ }^{\mathrm{b}}$ Individuals randomized to vortioxetine 15 and $20 \mathrm{mg}$ received vortioxetine $10 \mathrm{mg}$ for the first week, and those randomized to duloxetine $60 \mathrm{mg}$ received duloxetine $30 \mathrm{mg}$ for the first week. The assigned study dose was administered thereafter.

Patients receiving nontherapeutic doses of vortioxetine ( 1 and $2.5 \mathrm{mg} /$ day) were not included in any of the analyses. APTS - all patients treated set ( $N$ represents all randomized patients who took $\geq 1$ dose of study medication); CGI-S - Clinical Impressions-Severity of Illness; DSST - Digit Symbol Substitution Test; DUL - duloxetine; HAM-D $24-$ Hamilton Depression Rating Scale-24 item; MADRS - Montgomery-Åsberg Depression Rating Scale; MDE - major depressive episode; PBO - placebo; RAVLT - Rey Auditory Verbal Learning Test; VEN - venlafaxine XR; VOR - vortioxetine. 
TABLE 2. Demographic and baseline characteristics of patients aged $\geq 55$ years (APTS)

\begin{tabular}{|c|c|c|c|c|c|c|}
\hline & $\begin{array}{l}\text { Placebo } \\
(\mathrm{N}=561)\end{array}$ & $\begin{array}{l}\text { Vortioxetine } 5 \mathrm{mg} \\
\quad(\mathrm{n}=376)\end{array}$ & $\begin{array}{l}\text { Vortioxetine } 10 \mathrm{mg} \\
\quad(\mathrm{n}=259)\end{array}$ & $\begin{array}{l}\text { Vortioxetine } 15 \mathrm{mg} \\
\quad(\mathrm{n}=118)\end{array}$ & $\begin{array}{l}\text { Vortioxetine } 20 \mathrm{mg} \\
\quad(\mathrm{n}=194)\end{array}$ & $\begin{array}{l}\text { Vortioxetine all doses } \\
\qquad(\mathrm{N}=947)\end{array}$ \\
\hline \multicolumn{7}{|l|}{ Gender, $n(\%)$} \\
\hline Male & $202(36.0)$ & $121(32.2)$ & $91(35.1)$ & $37(31.4)$ & $63(32.5)$ & $312(32.9)$ \\
\hline Female & $359(64.0)$ & $255(67.8)$ & $168(64.9)$ & $81(68.6)$ & $131(67.5)$ & $635(67.1)$ \\
\hline \multicolumn{7}{|l|}{ Race, $n(\%)$} \\
\hline Caucasian & $516(92.0)$ & $337(89.6)$ & $227(87.6)$ & 109 (92.4) & $180(92.8)$ & $853(90.1)$ \\
\hline Asian & $11(2.0)$ & $17(4.5)$ & $16(6.2)$ & 0 & $2(1.0)$ & $35(3.7)$ \\
\hline Black or African American & $30(5.3)$ & $20(5.3)$ & $14(5.4)$ & $9(7.6)$ & $9(4.6)$ & $52(5.5)$ \\
\hline Other & $4(0.7)$ & $2(0.5)$ & $2(0.8)$ & 0 & $3(1.5)$ & $7(0.7)$ \\
\hline \multicolumn{7}{|l|}{ Region, $n(\%)$} \\
\hline US & $247(44.0)$ & $147(39.1)$ & $69(26.6)$ & $65(55.1)$ & $61(31.4)$ & $342(36.1)$ \\
\hline Non-US & $314(56.0)$ & $229(60.9)$ & $190(73.4)$ & $53(44.9)$ & $133(68.6)$ & $605(63.9)$ \\
\hline \multicolumn{7}{|l|}{ Age (years) } \\
\hline Mean (SD) & $62.8(6.33)$ & $64.8(6.81)$ & $59.9(4.25)$ & $61.7(5.38)$ & $60.4(4.46)$ & $62.1(5.98)$ \\
\hline Range & $55-85$ & $55-88$ & $55-75$ & $55-75$ & $55-75$ & $55-88$ \\
\hline$\geq 65, \mathrm{n}(\%)$ & $214(38.1)$ & $197(52.4)$ & $34(13.1)$ & $28(23.7)$ & $33(17.0)$ & $292(30.8)$ \\
\hline$\geq 75, n(\%)$ & $28(5.0)$ & $26(6.9)$ & $1(0.4)$ & $1(0.8)$ & $1(0.5)$ & $29(3.1)$ \\
\hline \multicolumn{7}{|l|}{ BMI $\left(\mathrm{kg} / \mathrm{m}^{2}\right)$} \\
\hline Mean (SD) & $28.53(5.95)$ & $28.25(6.25)$ & $27.79(5.72)$ & $28.92(6.53)$ & $27.61(4.94)$ & $28.08(5.90)$ \\
\hline Range & $15.6-58.5$ & $17.4-68.4$ & $16.7-54.6$ & $16.8-60.4$ & $16.0-43.2$ & $16.0-68.4$ \\
\hline$<25, \mathrm{n}(\%)$ & $165(29.4)$ & $118(31.4)$ & $85(32.8)$ & $37(31.4)$ & $63(32.5)$ & $303(32.0)$ \\
\hline $25-<30, n(\%)$ & $208(37.1)$ & $149(39.6)$ & $97(37.5)$ & $39(33.1)$ & $68(35.1)$ & $353(37.3)$ \\
\hline$\geq 30, n(\%)$ & $188(33.5)$ & $108(28.7)$ & $77(29.7)$ & $42(35.6)$ & $62(32.0)$ & $289(30.5)$ \\
\hline Missing & 0 & $1(0.3)$ & 0 & 0 & $1(0.5)$ & $2(0.2)$ \\
\hline \multicolumn{7}{|l|}{ Number of previous MDEs } \\
\hline 0 & $39(7.0)$ & $40(10.6)$ & $31(12.0)$ & 0 & $13(6.7)$ & $84(8.9)$ \\
\hline $1-3$ & $378(67.4)$ & $237(63.0)$ & $154(59.5)$ & $78(66.1)$ & $123(63.4)$ & $592(62.5)$ \\
\hline $4-6$ & $106(18.9)$ & $78(20.7)$ & $58(22.4)$ & $35(29.7)$ & $42(21.6)$ & $213(22.5)$ \\
\hline$>6$ & $38(6.8)$ & $21(5.6)$ & $16(6.2)$ & $5(4.2)$ & $16(8.2)$ & $58(6.1)$ \\
\hline \multicolumn{7}{|l|}{ Length of current MDE (weeks) } \\
\hline Median & 24.0 & 22.5 & 21.0 & 24.5 & 26.0 & 23.0 \\
\hline Range & $4-884$ & $4-780$ & $12-1138$ & $13-212$ & $13-216$ & $4-1138$ \\
\hline$<24$ weeks, n (\%) & $271(48.3)$ & $194(51.6)$ & $141(54.4)$ & $57(48.3)$ & $89(45.9)$ & $481(50.8)$ \\
\hline$\geq 24$ weeks, n (\%) & $290(51.7)$ & $182(48.4)$ & $117(45.2)$ & $61(51.7)$ & $105(54.1)$ & $465(49.1)$ \\
\hline Missing & 0 & 0 & $1(0.4)$ & 0 & 0 & $1(0.1)$ \\
\hline \multicolumn{7}{|l|}{ MADRS total score * } \\
\hline $\mathrm{N}$ & 555 & 375 & 258 & 118 & 193 & 944 \\
\hline Mean (SD) & $31.6(3.92)$ & $31.6(4.05)$ & $32.1(3.64)$ & $32.2(4.14)$ & $31.5(3.68)$ & $31.8(3.88)$ \\
\hline \multicolumn{7}{|l|}{ CGI-S score * } \\
\hline $\mathrm{N}$ & 555 & 375 & 258 & 118 & 193 & 944 \\
\hline Mean (SD) & $4.7(0.68)$ & $4.8(0.73)$ & $4.8(0.65)$ & $4.7(0.62)$ & $4.6(0.61)$ & $4.7(0.68)$ \\
\hline
\end{tabular}

unstructured covariance matrix and included terms for center, visit, treatment, and baseline score by visit interaction, and treatment by visit interaction. For MADRS response and MADRS remission, logistic regression using LOCF adjusting for baseline MADRS score was used to provide ORs as input for the meta-analysis.

Results represent the least squares (LS) mean differences or ORs versus placebo with $95 \%$ CIs. All statistical tests were 2-sided with a 0.05 significance level. Standardized effect sizes (SES) were then calculated for the difference from placebo in change from baseline and interpreted as Cohen's $d$ statistics.
Safety and baseline characteristics were examined using pooled data of the safety set (or all patients treated set [APTS]), which includes all randomized patients who took at least 1 dose of study medication. Demographics and TEAEs were summarized with descriptive statistics. The most frequently reported TEAEs, concurrent medical conditions, and concomitant medications (occurring in or used by $\geq 5 \%$ of patients in any treatment arm) are also listed. The number and percentage of individuals experiencing potentially clinically significant ECG or liver enzyme value deviations are reported. Vital signs and body weight were 
TABLE 3. Most frequently reported ( $\geq 5 \%$ in any treatment arm) concurrent medical conditions by preferred term in patients aged $\geq 55$ years

\begin{tabular}{lcccccc}
$\begin{array}{l}\text { MedDRA preferred term, patients, } \\
\mathrm{n}(\%)\end{array}$ & $\begin{array}{c}\text { Placebo } \\
(\mathrm{N}=561)\end{array}$ & $\begin{array}{c}\text { Vortioxetine } 5 \mathrm{mg} \\
(\mathrm{n}=376)\end{array}$ & $\begin{array}{c}\text { Vortioxetine } 10 \mathrm{mg} \\
(\mathrm{n}=259)\end{array}$ & $\begin{array}{c}\text { Vortioxetine } 15 \mathrm{mg} \\
(\mathrm{n}=118)\end{array}$ & $\begin{array}{c}\text { Vortioxetine 20 } \mathrm{mg} \\
(\mathrm{n}=194)\end{array}$ & $\begin{array}{c}\text { Vortioxetine all doses } \\
(\mathrm{N}=947)\end{array}$ \\
\hline $\begin{array}{l}\text { Patients with any concurrent } \\
\quad \text { medical conditions }\end{array}$ & $469(83.6)$ & $330(87.8)$ & $211(81.5)$ & $103(87.3)$ & $157(80.9)$ & $801(84.6)$ \\
$\begin{array}{l}\text { Hypertension } \\
\text { Postmenopause }\end{array}$ & $189(33.7)$ & $119(31.6)$ & $70(27.0)$ & $36(30.5)$ & $59(30.4)$ & $284(30.0)$ \\
Hypercholesterolemia & $53(9.4)$ & $52(13.8)$ & $31(12.0)$ & $19(16.1)$ & $19(9.8)$ & $121(12.8)$ \\
Menopause & $84(15.0)$ & $58(15.4)$ & $27(10.4)$ & $13(11.0)$ & $22(11.3)$ & $120(12.7)$ \\
Osteoarthritis & $62(11.1)$ & $43(11.4)$ & $50(19.3)$ & $6(5.1)$ & $18(9.3)$ & $117(12.4)$ \\
Hypothyroidism & $53(9.4)$ & $38(10.1)$ & $21(8.1)$ & $13(11.0)$ & $15(7.7)$ & $87(9.2)$ \\
Insomnia & $40(7.1)$ & $30(8.0)$ & $23(8.9)$ & $11(9.3)$ & $16(8.2)$ & $80(8.4)$ \\
Headache & $47(8.4)$ & $36(9.6)$ & $16(6.2)$ & $9(7.6)$ & $11(5.7)$ & $72(7.6)$ \\
Back pain & $37(6.6)$ & $31(8.2)$ & $8(3.1)$ & $11(9.3)$ & $16(8.2)$ & $66(7.0)$ \\
Drug hypersensitivity & $42(7.5)$ & $21(5.6)$ & $18(6.9)$ & $8(6.8)$ & $13(6.7)$ & $60(6.3)$ \\
Seasonal allergy & $33(5.9)$ & $18(4.8)$ & $15(5.8)$ & $8(6.8)$ & $10(5.2)$ & $51(5.4)$ \\
Gastroesophageal reflux disease & $49(8.7)$ & $12(3.2)$ & $12(4.6)$ & $10(8.5)$ & $15(7.7)$ & $49(5.2)$ \\
Asthma & $37(6.6)$ & $18(4.8)$ & $6(2.3)$ & $11(9.3)$ & $13(6.7)$ & $48(5.1)$ \\
Obesity & $28(5.0)$ & $15(4.0)$ & $11(4.2)$ & $8(6.8)$ & $12(6.2)$ & $46(4.9)$ \\
Hyperlipidemia & $33(5.9)$ & $24(6.4)$ & $11(4.2)$ & $4(3.4)$ & $7(3.6)$ & $46(4.9)$ \\
Type 2 diabetes mellitus & $22(3.9)$ & $14(3.7)$ & $12(4.6)$ & $6(5.1)$ & $8(4.1)$ & $40(4.2)$ \\
Arthritis & $22(3.9)$ & $23(6.1)$ & $5(1.9)$ & $2(1.7)$ & $8(4.1)$ & $38(4.0)$ \\
Osteoporosis & $21(3.7)$ & $20(5.3)$ & $5(1.9)$ & $5(4.2)$ & $5(2.6)$ & $35(3.7)$ \\
Blood cholesterol increased & $17(3.0)$ & $15(4.0)$ & $2(0.8)$ & $6(5.1)$ & $3(1.5)$ & $26(2.7)$ \\
Hypermetropia & $14(2.5)$ & $9(2.4)$ & $1(0.4)$ & $6(5.1)$ & $4(2.1)$ & $20(2.1)$ \\
P & $10(1.8)$ & $4(1.1)$ & $5(1.9)$ & $6(5.1)$ & $2(1.0)$ & $17(1.8)$ \\
\hline
\end{tabular}

Includes concurrent medical conditions that could have started before or during study treatment. MedDRA preferred terms are sorted in descending order based on their incidence in all patients receiving vortioxetine, regardless of dose. MedDRA - Medical Dictionary for Regulatory Activities, v14.1.

TABLE 4. Most frequently reported ( $\geq 5 \%$ in any treatment arm) concomitant medications by therapeutic class in patients aged $\geq 55$ years (APTS)

\begin{tabular}{|c|c|c|c|c|c|c|}
\hline Therapeutic class, patients, n (\%) & $\begin{array}{l}\text { Placebo } \\
(\mathrm{N}=561)\end{array}$ & $\begin{array}{l}\text { Vortioxetine } 5 \mathrm{mg} \\
\qquad(\mathrm{n}=376)\end{array}$ & $\begin{array}{l}\text { Vortioxetine } 10 \mathrm{mg} \\
\quad(\mathrm{n}=259)\end{array}$ & $\begin{array}{l}\text { Vortioxetine } 15 \mathrm{mg} \\
\quad(\mathrm{n}=118)\end{array}$ & $\begin{array}{l}\text { Vortioxetine } 20 \mathrm{mg} \\
\quad(\mathrm{n}=194)\end{array}$ & $\begin{array}{l}\text { Vortioxetine all doses } \\
\qquad(\mathrm{N}=947)\end{array}$ \\
\hline $\begin{array}{l}\text { Patients with any concomitant } \\
\text { medications }\end{array}$ & $390(69.5)$ & $274(72.9)$ & $166(64.1)$ & $90(76.3)$ & $131(67.5)$ & $661(69.8)$ \\
\hline ACE inhibitors & $116(20.7)$ & $72(19.1)$ & $44(17.0)$ & $24(20.3)$ & $37(19.1)$ & $177(18.7)$ \\
\hline Lipid-modifying agents & $107(19.1)$ & $74(19.7)$ & $33(12.7)$ & $19(16.1)$ & $34(17.5)$ & $160(16.9)$ \\
\hline Analgesics & $95(16.9)$ & $59(15.7)$ & $27(10.4)$ & $23(19.5)$ & $25(12.9)$ & $134(14.1)$ \\
\hline $\begin{array}{l}\text { Anti-inflammatory and anti-rheumatic } \\
\text { products }\end{array}$ & $70(12.5)$ & $49(13.0)$ & $29(11.2)$ & $26(22.0)$ & $26(13.4)$ & $130(13.7)$ \\
\hline Vitamins & $69(12.3)$ & $40(10.6)$ & $21(8.1)$ & $14(11.9)$ & $25(12.9)$ & $100(10.6)$ \\
\hline Drugs for acid-related disorders & $52(9.3)$ & $37(9.8)$ & $22(8.5)$ & $13(11.0)$ & $19(9.8)$ & $91(9.6)$ \\
\hline Thyroid therapy & $47(8.4)$ & $37(9.8)$ & $26(10.0)$ & $11(9.3)$ & $14(7.2)$ & $88(9.3)$ \\
\hline Beta-blocking agents & $63(11.2)$ & $40(10.6)$ & $22(8.5)$ & $5(4.2)$ & $20(10.3)$ & $87(9.2)$ \\
\hline Neuroleptics & $35(6.2)$ & $36(9.6)$ & $15(5.8)$ & $3(2.5)$ & $12(6.2)$ & $66(7.0)$ \\
\hline Calcium channel blockers & $39(7.0)$ & $31(8.2)$ & $15(5.8)$ & $9(7.6)$ & $9(4.6)$ & $64(6.8)$ \\
\hline $\begin{array}{l}\text { Sex hormones and modulators of the } \\
\text { genital system }\end{array}$ & $33(5.9)$ & $20(5.3)$ & $20(7.7)$ & $6(5.1)$ & $10(5.2)$ & $56(5.9)$ \\
\hline Drugs used in diabetes & $24(4.3)$ & $29(7.7)$ & $12(4.6)$ & $3(2.5)$ & $11(5.7)$ & $55(5.8)$ \\
\hline Diuretics & $39(7.0)$ & $22(5.9)$ & $12(4.6)$ & $8(6.8)$ & $12(6.2)$ & $54(5.7)$ \\
\hline Drugs for obstructive airway diseases & $33(5.9)$ & $20(5.3)$ & $13(5.0)$ & $6(5.1)$ & $13(6.7)$ & $52(5.5)$ \\
\hline Mineral supplements & $40(7.1)$ & $24(6.4)$ & $11(4.2)$ & $9(7.6)$ & $8(4.1)$ & $52(5.5)$ \\
\hline Antibacterials for systemic use & $33(5.9)$ & $29(7.7)$ & $10(3.9)$ & $6(5.1)$ & $5(2.6)$ & $50(5.3)$ \\
\hline Antihistamines for systemic use & $28(5.0)$ & $12(3.2)$ & $10(3.9)$ & $5(4.2)$ & $14(7.2)$ & $41(4.3)$ \\
\hline Antithrombotic agents & $36(6.4)$ & $31(8.2)$ & $5(1.9)$ & $4(3.4)$ & 0 & $40(4.2)$ \\
\hline Urologicals & $16(2.9)$ & $22(5.9)$ & $7(2.7)$ & $3(2.5)$ & $2(1.0)$ & $34(3.6)$ \\
\hline
\end{tabular}

ACE - angiotensin-converting enzyme; APTS - all patients treated set ( $N$ represents all randomized patients who took $\geq 1$ dose of study medication). Includes concomitant medications that could have started before or during study treatment. Therapeutic classes are sorted in descending order based on their incidence in all patients receiving vortioxetine, regardless of dose. 


\begin{tabular}{|c|c|c|c|c|c|c|}
\hline NCT Identifier & $\begin{array}{l}\text { VOR } \\
\text { dose }\end{array}$ & $\begin{array}{c}\text { MADRS Total Score } \\
\text { Difference from Placebo }(95 \% \mathrm{Cl})\end{array}$ & $\mathbf{N}$ & $\begin{array}{l}\text { Difference } \\
\quad( \pm \mathrm{SE})\end{array}$ & $\begin{array}{l}\text { Standardized } \\
\text { Effect Size }\end{array}$ & $P$-value \\
\hline \multirow[t]{2}{*}{ NCT00839423 } & $5 \mathrm{mg}$ & $\longmapsto$ & 19 & $-6.60(2.67)$ & -0.87 & 0.017 \\
\hline & $10 \mathrm{mg}$ & $\longmapsto \square$ & 22 & $-7.35(2.54)$ & -0.99 & 0.006 \\
\hline \multirow[t]{2}{*}{ NCT00635219 } & $5 \mathrm{mg}$ & $\longrightarrow$ & 26 & $-0.98(2.56)$ & -0.11 & 0.703 \\
\hline & $10 \mathrm{mg}$ & $\longrightarrow$ & 32 & $-0.59(2.51)$ & -0.06 & 0.814 \\
\hline \multirow[t]{2}{*}{ NCT00735709 } & $5 \mathrm{mg}$ & $\longmapsto \diamond$ & 36 & $-5.84(1.74)$ & -0.77 & 0.001 \\
\hline & $10 \mathrm{mg}$ & $\longmapsto \square-$ & 32 & $-5.46(1.76)$ & -0.73 & 0.002 \\
\hline \multirow[t]{2}{*}{ NCT01140906 } & $15 \mathrm{mg}$ & $\longmapsto \Delta$ & 40 & $-4.61(1.68)$ & -0.60 & 0.007 \\
\hline & $20 \mathrm{mg}$ & $\longmapsto 0-1$ & 32 & $-7.02(1.80)$ & -0.90 & $<0.001$ \\
\hline \multirow[t]{2}{*}{ NCT01153009 } & $15 \mathrm{mg}$ & $\triangle$ & 18 & $-3.69(2.96)$ & -0.40 & 0.217 \\
\hline & $20 \mathrm{mg}$ & $\mathrm{O}$ & 15 & $-6.26(3.22)$ & -0.66 & 0.056 \\
\hline \multirow[t]{2}{*}{ NCT01163266 } & $10 \mathrm{mg}$ & & 25 & $-1.96(2.69)$ & -0.20 & 0.468 \\
\hline & $20 \mathrm{mg}$ & & 29 & $-0.78(2.63)$ & -0.08 & 0.767 \\
\hline NCT00672958 & $5 \mathrm{mg}$ & & 49 & $0.64(2.22)$ & 0.06 & 0.775 \\
\hline NCT00672620 & $5 \mathrm{mg}$ & 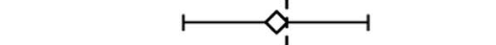 & 27 & $-0.50(2.19)$ & -0.06 & 0.821 \\
\hline \multirow[t]{2}{*}{ NCT01179516 } & $10 \mathrm{mg}$ & i & 27 & $1.69(2.57)$ & 0.17 & 0.512 \\
\hline & $15 \mathrm{mg}$ & $\Delta$ & 32 & $4.54(2.44)$ & 0.45 & 0.067 \\
\hline \multirow[t]{3}{*}{ NCT01255787 } & $5 \mathrm{mg}$ & $\longrightarrow$ & 30 & $1.77(2.38)$ & 0.19 & 0.460 \\
\hline & $10 \mathrm{mg}$ & 1 & 34 & $-1.71(2.31)$ & -0.18 & 0.462 \\
\hline & $20 \mathrm{mg}$ & $\longrightarrow$ & 33 & $0.42(2.33)$ & 0.05 & 0.857 \\
\hline NCT00811252 & $5 \mathrm{mg}$ & $\longmapsto \diamond$ & 137 & $-4.74(1.04)$ & -0.56 & $<0.001$ \\
\hline \multirow[t]{2}{*}{ NCT01422213 } & $10 \mathrm{mg}$ & $\longmapsto \square$ & 50 & $-3.16(1.70)$ & -0.37 & 0.065 \\
\hline & $20 \mathrm{mg}$ & $\longmapsto-0-1$ & 56 & $-8.62(1.67)$ & -1.00 & $<0.001$ \\
\hline \multirow[t]{6}{*}{ Meta-analysis } & $5 \mathrm{mg}$ & $-i_{1}^{\prime}$ & 324 & $-2.56(1.22)$ & -0.17 & 0.035 \\
\hline & $10 \mathrm{mg}$ & $\mapsto \square-i$ & 222 & $-2.87(1.07)$ & -0.25 & 0.007 \\
\hline & $15 \mathrm{mg}$ & $\Delta i$ & 90 & $-1.32(2.99)$ & -0.06 & 0.658 \\
\hline & $20 \mathrm{mg}$ & $\longmapsto-1$ & 165 & $-4.65(1.86)$ & -0.27 & 0.012 \\
\hline & -15 & $\begin{array}{llll}-12 & -9 & -6 & -3\end{array}$ & 12 & & & \\
\hline & & $\begin{array}{l}\leftarrow \text { Better } \\
\text { than placebo }\end{array}$ & & & & \\
\hline
\end{tabular}

\section{VOR - vortioxetine.}

FIGURE 1. Difference from placebo in MADRS total score change from baseline to study endpoint in patients aged $\geq 55$ years (FAS, MMRM).

summarized by the mean change from baseline at study endpoint, as well as potentially clinically significant values during the treatment period.

\section{Results}

\section{Patients}

The subpopulation included in the meta-analysis comprised 1508 patients aged 55 years or older $(27.8 \%$ of all patients) who were treated with placebo $(\mathrm{N}=561)$ or vortioxetine $5-20 \mathrm{mg} /$ day $(\mathrm{N}=947)$. The demographic and baseline characteristics were similar across all treatment groups (Table 2). Patients were predominantly women (placebo, 64.0\%; vortioxetine $5-20 \mathrm{mg} /$ day, $64.9-68.6 \%)$ and white $(92.0 \% ; 87.6-92.8 \%)$, with ages ranging from 55 to 88 years (mean age: placebo, 62.8 years; vortioxetine $5-20 \mathrm{mg} /$ day, $59.9-64.8$ years). The proportion of patients outside of the US was higher than the proportion of patients within the US, with the exception of vortioxetine $15 \mathrm{mg} /$ day (Table 2).

The majority of patients in this post hoc meta-analysis had concurrent medical conditions, with the most common (incidence $\geq 10 \%$ in any treatment arm) conditions being hypertension, menopause/postmenopause, hypercholesterolemia, and osteoarthritis (Table 3).

Many patients also took concomitant medications, with the most common (incidence $\geq 10 \%$ in any treatment arm) therapeutic classes being angiotensin-converting enzyme (ACE) inhibitors, lipid-modifying agents, analgesics, anti-inflammatory and anti-rheumatic products, 


\begin{tabular}{|c|c|c|c|c|c|}
\hline MADRS Item & $\begin{array}{l}\text { VOR } \\
\text { dose }\end{array}$ & $\begin{array}{c}\text { MADRS Item Score } \\
\text { Difference from Placebo }(95 \% \mathrm{Cl})\end{array}$ & $\mathbf{N}$ & $\begin{array}{l}\text { Difference } \\
\quad( \pm S E)\end{array}$ & $P$-value \\
\hline \multirow{4}{*}{$\begin{array}{l}\text { Item 4: } \\
\text { Reduced sleep }\end{array}$} & $5 \mathrm{mg}$ & $\longmapsto \diamond 1$ & 324 & $-0.25(0.21)$ & 0.225 \\
\hline & $10 \mathrm{mg}$ & $\longmapsto \square-1$ & 222 & $-0.42(0.16)$ & 0.008 \\
\hline & $15 \mathrm{mg}$ & $\longmapsto \nabla_{i}$ & 90 & $-0.09(0.32)$ & 0.768 \\
\hline & $20 \mathrm{mg}$ & $\longmapsto \square \longrightarrow$ & 165 & $-0.52(0.25)$ & 0.037 \\
\hline \multirow{4}{*}{$\begin{array}{l}\text { Item 5: } \\
\text { Reduced appetite }\end{array}$} & $5 \mathrm{mg}$ & 만 & 324 & $-0.06(0.10)$ & 0.552 \\
\hline & $10 \mathrm{mg}$ & $\mapsto \Delta$ & 222 & $-0.18(0.10)$ & 0.065 \\
\hline & $15 \mathrm{mg}$ & $10-4$ & 90 & $-0.26(0.16)$ & 0.098 \\
\hline & $20 \mathrm{mg}$ & $\longmapsto \Delta \mathrm{i}$ & 165 & $-0.26(0.18)$ & 0.150 \\
\hline \multirow{4}{*}{$\begin{array}{l}\text { Item 6: } \\
\text { Concentration } \\
\text { difficulties }\end{array}$} & $5 \mathrm{mg}$ & $\mapsto \square-1$ & 324 & $-0.28(0.14)$ & 0.039 \\
\hline & $10 \mathrm{mg}$ & 10 & 172 & $-0.28(0.19)$ & 0.145 \\
\hline & $15 \mathrm{mg}$ & 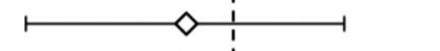 & 90 & $-0.27(0.47)$ & 0.558 \\
\hline & $20 \mathrm{mg}$ & 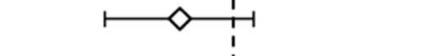 & 109 & $-0.31(0.22)$ & 0.157 \\
\hline \multirow{6}{*}{$\begin{array}{l}\text { Item 7: } \\
\text { Lassitude }\end{array}$} & $5 \mathrm{mg}$ & $\longmapsto \Delta:$ & 324 & $-0.23(0.18)$ & 0.207 \\
\hline & $10 \mathrm{mg}$ & 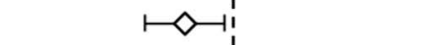 & 222 & $-0.28(0.12)$ & 0.017 \\
\hline & $15 \mathrm{mg}$ & : & 90 & $0.12(0.46)$ & 0.795 \\
\hline & $20 \mathrm{mg}$ & $\longmapsto-1 \longrightarrow$ & 165 & $-0.60(0.19)$ & 0.002 \\
\hline & -2 & -1 & 2 & & \\
\hline & & $\begin{array}{l}\text { Worse } \rightarrow \\
\text { than placebo }\end{array}$ & & & \\
\hline
\end{tabular}

FIGURE 2. Difference from placebo in select MADRS individual item scores change from baseline to study endpoint in patients aged $\geq 55$ years (FAS, MMRM).

vitamins, drugs for acid-related disorders, thyroid therapy, and beta-blocking agents (Table 4).

\section{Efficacy}

Based on this meta-analysis, the difference versus placebo in change from baseline on MADRS total score was statistically significantly in favor of vortioxetine $5 \mathrm{mg}(\mathrm{N}=324$, $\Delta-2.56 ; \quad P=0.035), \quad 10 \mathrm{mg} \quad(\mathrm{N}=222, \quad \Delta-2.87$; $P=0.007)$, and $20 \mathrm{mg}(\mathrm{N}=165, \Delta-4.65 ; P=0.012)$, whereas vortioxetine $15 \mathrm{mg}$ was not statistically significant versus placebo $(\mathrm{N}=90, \Delta-1.32 ; P=0.658)$ (Figure 1$)$.

When the difference from placebo in change from baseline in select MADRS individual item scores was analyzed, sleep was significantly improved with vortioxetine $10 \mathrm{mg}$ and $20 \mathrm{mg} \quad(P=0.008$ and $P=0.037$, respectively); concentration was significantly improved with vortioxetine $5 \mathrm{mg}(P=0.039)$; and lassitude was significantly improved with vortioxetine $10 \mathrm{mg}$ and $20 \mathrm{mg}$ $(P=0.017$ and $P=0.002$, respectively) (Figure 2$)$. Appetite was not significantly improved at any vortioxetine dose.

The meta-analysis of treatment response rates $(\geq 50 \%$ decrease from baseline in MADRS total score) demonstrated a benefit versus placebo for patients treated with vortioxetine (Figure 3). Statistically significant differences in response rates compared with placebo were observed for vortioxetine $10 \mathrm{mg}(47.9 \%, P=0.002$; OR 1.82) and $20 \mathrm{mg}$ (54.9\%, $P<0.001$; OR 2.46). The rate of treatment response for placebo in this population was $35.5 \%$.

Remission rates (MADRS total score $\leq 10$ ) at study endpoint demonstrated a statistically significant benefit only for patients treated with vortioxetine $20 \mathrm{mg}(37.3 \%$, $P<0.001$; OR 2.71) (Figure 4). The rate of remission for placebo in this population was $20.2 \%$.

\section{Safety and tolerability}

The incidence of TEAEs was $61.5 \%$ for placebo. Among individuals receiving vortioxetine $5-20 \mathrm{mg} /$ day, $62.3 \%$ reported TEAEs, and the incidence did not increase with vortioxetine dose (Table 5). Compared with placebo, the incidence of treatment-related adverse events was higher for all vortioxetine doses. Rates of discontinuation due to TEAEs were similar to placebo $(5.2 \%)$ in the vortioxetine $5 \mathrm{mg}(5.1 \%)$ and $10 \mathrm{mg}(5.0 \%)$ groups, but were higher at higher doses $(12.7 \%$ with vortioxetine $15 \mathrm{mg}$, and $9.8 \%$ with $20 \mathrm{mg}$ ). The incidence of serious TEAEs was low and similar to placebo across all vortioxetine doses, with no increase at the higher vortioxetine doses of 10 to $20 \mathrm{mg} /$ day (Table 5). A 74-year-old woman with a medical history of cholelithiasis and treated with vortioxetine $5 \mathrm{mg}$ died from 


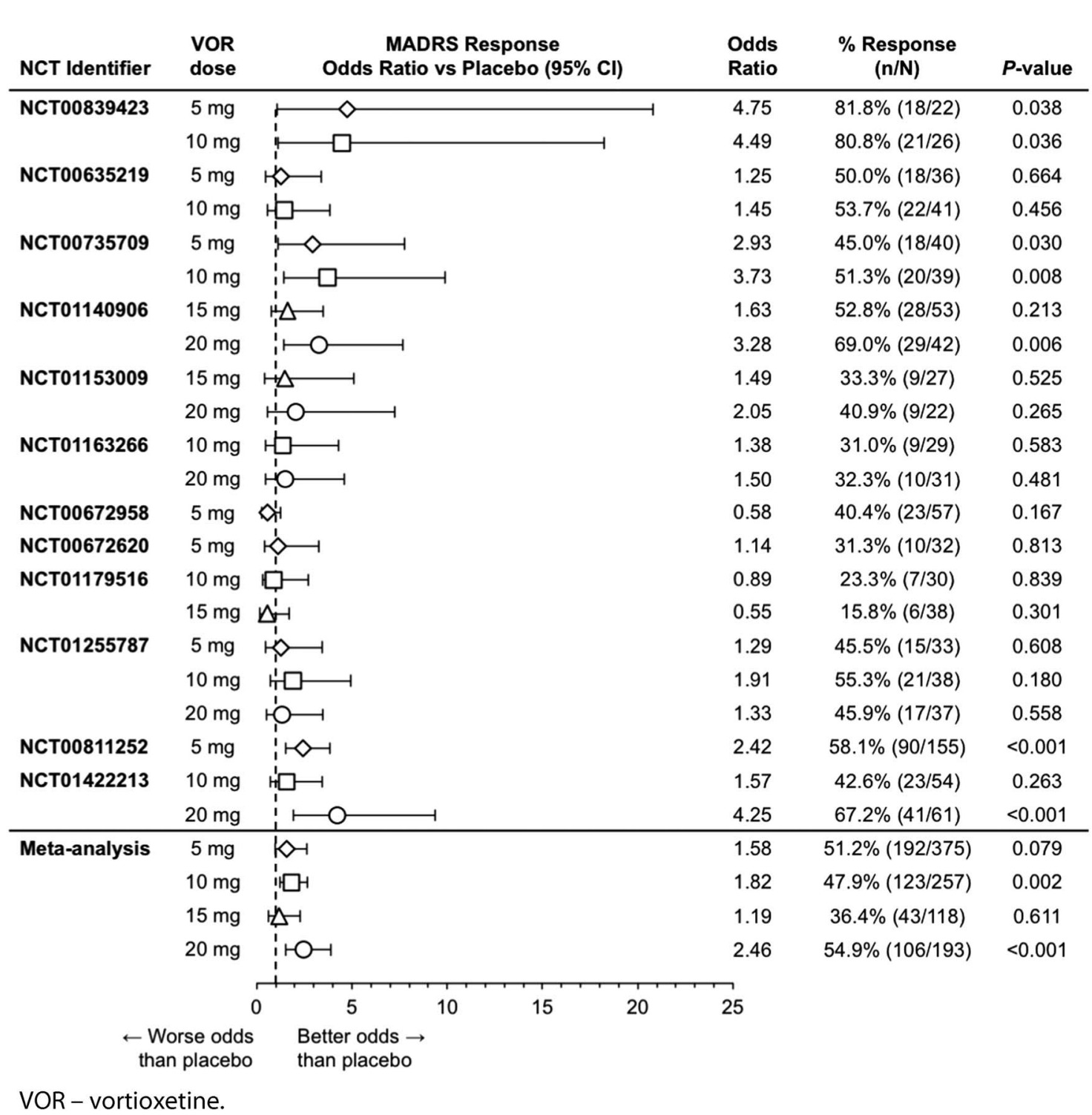

FIGURE 3. Percentage (\%) of responding patients aged $\geq 55$ years (defined as $\geq 50 \%$ decrease from baseline in MADRS total score) at study endpoint (FAS, LOCF).

gall bladder cancer approximately 1 month after withdrawal from the study (NCT00635219).

The safety profile of vortioxetine in this pooled analysis was similar to what was observed in the individual trials. ${ }^{30,34-44}$ The incidence of specific TEAEs was similar across treatment arms, except for nausea (which was more than twice as high for all individual vortioxetine doses than for placebo), vomiting (more than twice as high for vortioxetine $10-20 \mathrm{mg}$ than for placebo), constipation (more than twice as high for vortioxetine $15 \mathrm{mg}$ than for placebo), and anxiety (more than twice as high for vortioxetine $15 \mathrm{mg}$ than for placebo) (Table 6).

There were no clinically relevant changes in vital signs values or body weight after 6-8 weeks of treatment with vortioxetine (Table 7). The incidence of ECG values and liver enzyme levels of potential clinical significance were similar to placebo for all vortioxetine doses.

\section{Discussion}

We undertook a meta-analysis that provides evidence that vortioxetine $5-20 \mathrm{mg}$ /day is efficacious and well tolerated in patients with MDD aged 55 years or older. The metaanalysis of clinical efficacy suggests a dose-response relationship with vortioxetine $5 \mathrm{mg}, 10 \mathrm{mg}$, and $20 \mathrm{mg}$, as demonstrated by the percentage of patients who responded to treatment. Additionally, given the challenge of interpreting any potential predictors of response in a highly heterogeneous population of patients with MDD, this analysis yielded a similar result to that seen to date in the overall vortioxetine clinical program. ${ }^{30,34-44}$ Identifying predictors of response to vortioxetine in MDD does, however, remain an important and worthwhile endeavor. Because vortioxetine $15 \mathrm{mg}$ was only assessed in 3 of the 12 trials included here, this treatment group had the 


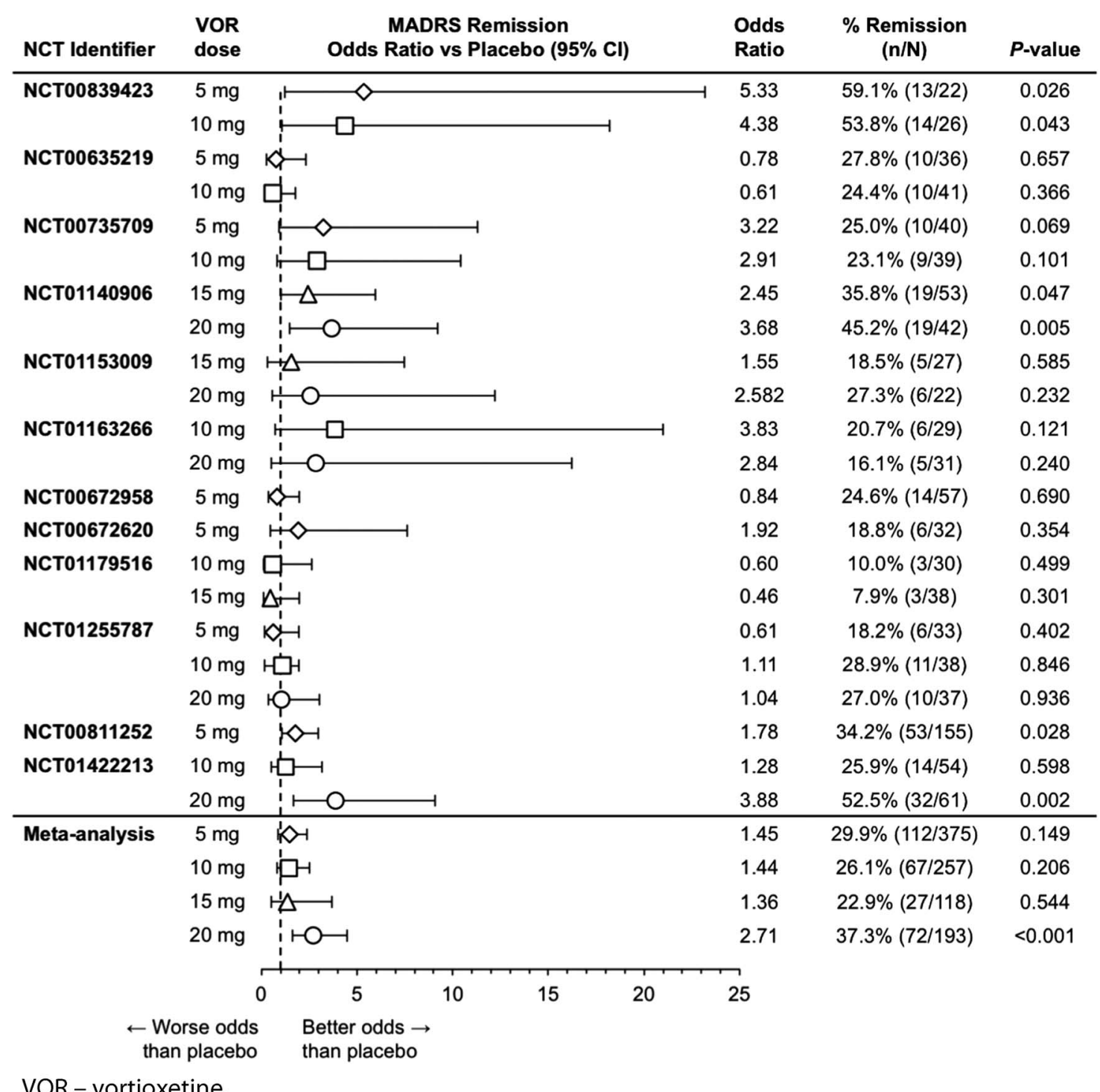

FIGURE 4. Percentage (\%) of remitting patients aged $\geq 55$ years (defined as MADRS $\leq 10$ ) at study endpoint (FAS, LOCF).

\begin{tabular}{|c|c|c|c|c|c|c|}
\hline Patients, n (\%) & $\begin{array}{l}\text { Placebo } \\
(\mathrm{N}=561)\end{array}$ & $\begin{array}{l}\text { Vortioxetine } 5 \mathrm{mg} \\
\quad(n=376)\end{array}$ & $\begin{array}{l}\text { Vortioxetine } 10 \mathrm{mg} \\
\quad(\mathrm{n}=259)\end{array}$ & $\begin{array}{l}\text { Vortioxetine } 15 \mathrm{mg} \\
\quad(\mathrm{n}=118)\end{array}$ & $\begin{array}{l}\text { Vortioxetine } 20 \mathrm{mg} \\
\quad(\mathrm{n}=194)\end{array}$ & $\begin{array}{l}\text { Vortioxetine all doses } \\
\qquad(\mathrm{N}=947)\end{array}$ \\
\hline Any TEAEs & $345(61.5)$ & $237(63.0)$ & $146(56.4)$ & $80(67.8)$ & $127(65.5)$ & $590(62.3)$ \\
\hline Treatment-related AEs & $259(46.2)$ & $187(49.7)$ & $127(49.0)$ & $73(61.9)$ & $115(59.3)$ & $502(53.0)$ \\
\hline $\begin{array}{l}\text { TEAEs leading to study } \\
\text { discontinuation }\end{array}$ & $29(5.2)$ & $19(5.1)$ & $13(5.0)$ & $15(12.7)$ & $19(9.8)$ & $66(7.0)$ \\
\hline Serious TEAES & $7(1.2)$ & $8(2.1)$ & $2(0.8)$ & $1(0.8)$ & 0 & $11(1.2)$ \\
\hline Deaths & 0 & $1(0.3)$ * & 0 & 0 & 0 & $1(0.1)$ * \\
\hline
\end{tabular}

Includes adverse events occurring on or after the first dose of study treatment and within 30 days post dosing.

*A 74-year-old woman with a medical history of cholelithiasis and treated with vortioxetine $5 \mathrm{mg}$ died from gall bladder cancer approximately 1 month after withdrawal from the study (NCT00635219). AE - adverse event; APTS - all patients treated set ( $N$ represents all randomized patients who took $\geq 1$ dose of study medication). 


\begin{tabular}{|c|c|c|c|c|c|c|}
\hline $\begin{array}{l}\text { MedDRA preferred term, } \\
\text { patients, } n(\%)\end{array}$ & $\begin{array}{l}\text { Placebo } \\
(\mathrm{N}=561)\end{array}$ & $\begin{array}{l}\text { Vortioxetine } 5 \mathrm{mg} \\
\quad(n=376)\end{array}$ & $\begin{array}{l}\text { Vortioxetine } 10 \mathrm{mg} \\
\qquad(\mathrm{n}=259)\end{array}$ & $\begin{array}{l}\text { Vortioxetine } 15 \mathrm{mg} \\
\quad(\mathrm{n}=118)\end{array}$ & $\begin{array}{l}\text { Vortioxetine } 20 \mathrm{mg} \\
\qquad(\mathrm{n}=194)\end{array}$ & $\begin{array}{l}\text { Vortioxetine all doses } \\
\qquad(\mathrm{N}=947)\end{array}$ \\
\hline Nausea & $49(8.7)$ & $68(18.1)$ & $47(18.1)$ & $40(33.9)$ & $62(32.0)$ & $217(22.9)$ \\
\hline Headache & 77 (13.7) & $44(11.7)$ & 30 (11.6) & $20(16.9)$ & 26 (13.4) & $120(12.7)$ \\
\hline Diarrhea & $33(5.9)$ & $21(5.6)$ & $14(5.4)$ & $13(11.0)$ & $14(7.2)$ & $62(6.5)$ \\
\hline Dizziness & $42(7.5)$ & $24(6.4)$ & $13(5.0)$ & $10(8.5)$ & $10(5.2)$ & $57(6.0)$ \\
\hline Dry mouth & $26(4.6)$ & $23(6.1)$ & $9(3.5)$ & $8(6.8)$ & $12(6.2)$ & $52(5.5)$ \\
\hline Constipation & $17(3.0)$ & $14(3.7)$ & $10(3.9)$ & $8(6.8)$ & $10(5.2)$ & $42(4.4)$ \\
\hline Fatigue & $17(3.0)$ & $17(4.5)$ & $7(2.7)$ & $6(5.1)$ & $3(1.5)$ & $33(3.5)$ \\
\hline Vomiting & $6(1.1)$ & $7(1.9)$ & $8(3.1)$ & $8(6.8)$ & $8(4.1)$ & $31(3.3)$ \\
\hline Anxiety & $8(1.4)$ & $3(0.8)$ & $3(1.2)$ & $6(5.1)$ & 0 & $12(1.3)$ \\
\hline
\end{tabular}

Includes adverse events occurring on or after the first dose and within 30 days post dosing. MedDRA preferred terms are sorted in descending order based on their incidence in all patients receiving vortioxetine, regardless of dose. MedDRA - Medical Dictionary for Regulatory Activities, v14.1. APTS - all patients treated set ( $N$ represents all randomized patients who took $\geq 1$ dose of study medication)

smallest sample size, resulting in substantially wider confidence intervals compared to the other doses. In addition, 2 of the 3 studies were focused in the US, whereas the last study was focused primarily in Europe. These discrepancies may contribute to the reasons why results from this group were not consistent with the dosedependent efficacy observed for the other doses, which has also been observed in other meta-analyses of vortioxetine studies. ${ }^{53-55}$ Such discrepancies ${ }^{56,57}$ are commonly seen in meta-analyses due to the heterogeneous effect among studies. ${ }^{58,59}$

Vortioxetine treatment demonstrated significant improvement in change from baseline of specific items on the MADRS associated with disability in patients with MDD. A study by Anderson et $a l^{52}$ found that in patients aged 60 years or older with at least 1 core symptom of an MDE (eg, depressed mood and loss of interest), there was a linear relationship between disability and a number of non-core symptoms (ie, sleep problems, appetite/weight change, psychomotor disturbance, and loss of energy). However, individual symptoms differentially affected the severity of the disability, with loss of energy related to the greatest decreases in cognitive functioning and increases in role dysfunction, number of disability days, number of health professional consultations, and nonspecific psychological stress. Accordingly, the corresponding items on the MADRS would be reduced sleep (Item 4), reduced appetite (Item 5), concentration difficulties (Item 6), and lassitude (Item 7), which were selected for specific analysis in this report. Treatment with vortioxetine 10 or $20 \mathrm{mg} /$ day significantly improved sleep and lassitude. Significant improvement in concentration was observed only in patients receiving vortioxetine $5 \mathrm{mg} /$ day.

Although the newer antidepressants are considered generally safe and well tolerated in elderly individuals, safety and tolerability profiles are heterogeneous. ${ }^{23}$ The safety assessment suggests that vortioxetine is well tolerated in this multi-morbid MDD population. Rates of serious AEs were similar to placebo and were not higher at vortioxetine doses of 10-20 mg/day, and rates of discontinuation due to AEs were similar to placebo in the vortioxetine $5 \mathrm{mg}$ and $10 \mathrm{mg}$ dosing groups. Although the patients aged 55 years or older included in this meta-analysis had a variety of comorbid medical conditions and were taking a variety of concomitant medications at baseline, there were no safety signals suggesting drug-drug interactions or worsening of preexisting conditions based on the $\mathrm{AE}$ profile as well as the low incidence of potentially clinically significant changes in vital signs and weight during the 6-8 weeks of treatment. Patients aged 55 and older are recommended to be initiated on vortioxetine in line with the overall adult population: patients are recommended to start on vortioxetine $10 \mathrm{mg} /$ day; however, patients may be initiated at a dose of $5 \mathrm{mg}$ daily if there are any concerns regarding tolerability.

\section{Limitations}

This study conducted meta-analyses of aggregated study-level data from 12 randomized, placebocontrolled trials, which have inherent heterogeneity. No direct comparison about the efficacy and safety of vortioxetine was made between the subpopulation aged 55 years or older and patients aged less than 55 years due to the unreliable estimation of age effects considering study heterogeneity. Patients with clinically significant psychiatric, psychotic, neurological, or neurodegenerative disorders or with clinically unstable illnesses were excluded from these randomized trials. 
TABLE 7. Potentially clinically significant values and shifts in vital signs, electrocardiograms, liver enzymes, and hody weight during treatment in patients aged $\geq 55$ years

\begin{tabular}{|c|c|c|c|c|c|c|}
\hline Patients, $\mathrm{n} / \mathrm{N}(\%)$ & $\begin{array}{c}\text { Placebo } \\
(\mathrm{N}=561)\end{array}$ & $\begin{array}{l}\text { Vortioxetine } 5 \mathrm{mg} \\
\quad(\mathrm{n}=376)\end{array}$ & $\begin{array}{c}\text { Vortioxetine } 10 \mathrm{mg} \\
\quad(\mathrm{n}=259)\end{array}$ & $\begin{array}{l}\text { Vortioxetine } 15 \mathrm{mg} \\
\quad(\mathrm{n}=118)\end{array}$ & $\begin{array}{l}\text { Vortioxetine } 20 \mathrm{mg} \\
\quad(\mathrm{n}=194)\end{array}$ & $\begin{array}{l}\text { Vortioxetine all doses } \\
\qquad(\mathrm{N}=947)\end{array}$ \\
\hline \multicolumn{7}{|l|}{ Vital signs } \\
\hline \multicolumn{7}{|l|}{ Standing systolic BP (mmHg) } \\
\hline Mean change (SD) * & $-1.9(13.56)$ & $-0.1(13.07)$ & $-0.5(12.09)$ & $-1.7(14.09)$ & $1.9(11.98)$ & $-0.1(12.80)$ \\
\hline$\leq 90$ and a decrease of $\geq 20$ & $1 / 524(0.2)$ & $5 / 342(1.5)$ & $4 / 220(1.8)$ & 2/117 (1.7) & $1 / 156(0.6)$ & $12 / 835(1.4)$ \\
\hline$\geq 180$ and an increase of $\geq 20$ & $7 / 524(1.3)$ & $3 / 342(0.9)$ & $1 / 220(0.5)$ & $1 / 117(0.9)$ & $0 / 156$ & $5 / 835(0.6)$ \\
\hline \multicolumn{7}{|l|}{ Standing diastolic BP (mmHg) } \\
\hline Mean change (SD) * & $-0.5(8.50)$ & $-0.9(8.44)$ & $-1.1(7.97)$ & $-0.8(8.44)$ & $0.7(7.93)$ & $-0.7(8.23)$ \\
\hline$\leq 50$ and a decrease of $\geq 15$ & $0 / 524$ & 0/342 & $1 / 220(0.5)$ & $0 / 117$ & 0/156 & $1 / 835(0.1)$ \\
\hline$\geq 105$ and an increase of $\geq 15$ & $9 / 524(1.7)$ & $3 / 342(0.9)$ & $2 / 220(0.9)$ & 2/117 (1.7) & $3 / 156(1.9)$ & $10 / 835(1.2)$ \\
\hline \multicolumn{7}{|l|}{ Standing pulse (bpm) } \\
\hline Mean change (SD) * & $-1.2(9.41)$ & $-0.8(9.65)$ & $-0.5(9.73)$ & $-0.4(9.13)$ & $0.3(9.34)$ & $-0.4(9.54)$ \\
\hline$\leq 50$ and a decrease of $\geq 15$ & $1 / 523(0.2)$ & $4 / 342(1.2)$ & $1 / 220(0.5)$ & $0 / 117$ & $0 / 156$ & $5 / 835(0.6)$ \\
\hline$\geq 120$ and an increase of $\geq 15$ & $0 / 523$ & $0 / 342$ & $0 / 220$ & $1 / 117(0.9)$ & $0 / 156$ & $1 / 835(0.1)$ \\
\hline \multicolumn{7}{|l|}{ Supine systolic BP (mmHg) } \\
\hline Mean change (SD) * & $-2.1(13.48)$ & $-0.8(11.72)$ & $-0.8(10.77)$ & $-1.5(12.82)$ & $1.2(11.96)$ & $-0.5(11.69)$ \\
\hline$\leq 90$ and a decrease of $\geq 20$ & $1 / 524(0.2)$ & $3 / 342(0.9)$ & $2 / 220(0.9)$ & $0 / 117$ & $0 / 156$ & $5 / 835(0.6)$ \\
\hline$\geq 180$ and an increase of $\geq 20$ & $8 / 524(1.5)$ & $4 / 342(1.2)$ & $1 / 220(0.5)$ & $1 / 117(0.9)$ & $0 / 156$ & $6 / 835(0.7)$ \\
\hline \multicolumn{7}{|l|}{ Supine diastolic BP (mmHg) } \\
\hline Mean change (SD) * & $-1.2(8.40)$ & $-1.4(8.22)$ & $-0.5(7.48)$ & $-1.6(9.10)$ & $1.0(6.43)$ & $-0.7(7.90)$ \\
\hline$\leq 50$ and a decrease of $\geq 15$ & $1 / 524(0.2)$ & $1 / 342(0.3)$ & $0 / 220$ & $0 / 117$ & $0 / 156$ & $1 / 835(0.1)$ \\
\hline$\geq 105$ and an increase of $\geq 15$ & $6 / 524(1.1)$ & $3 / 342(0.9)$ & $1 / 220(0.5)$ & $1 / 117(0.9)$ & $1 / 156(0.6)$ & $6 / 835(0.7)$ \\
\hline \multicolumn{7}{|l|}{ Supine pulse (bpm) } \\
\hline Mean change (SD) * & $-0.6(8.86)$ & $-1.2(8.59)$ & $-0.8(8.75)$ & $-1.6(9.12)$ & $-0.4(8.87)$ & $-1.0(8.75)$ \\
\hline$\leq 50$ and a decrease of $\geq 15$ & $2 / 524(0.4)$ & $4 / 342(1.2)$ & $4 / 220(1.8)$ & $0 / 117$ & $1 / 156(0.6)$ & $9 / 835(1.1)$ \\
\hline$\geq 120$ and an increase of $\geq 15$ & $0 / 524$ & $0 / 342$ & $0 / 220$ & $0 / 117$ & $0 / 156$ & $0 / 835$ \\
\hline \multicolumn{7}{|l|}{ Orthostatic systolic BP (mmHg) } \\
\hline Mean change (SD) * & $0.3(10.57)$ & $0.7(10.16)$ & $0.3(8.97)$ & $-0.2(10.20)$ & $0.7(11.24)$ & $0.5(10.07)$ \\
\hline Calculated decrease & & & & & & \\
\hline (standing-supine) $>40$ & $0 / 524$ & $1 / 342(0.3)$ & $2 / 220(0.9)$ & $0 / 117$ & $0 / 156$ & $3 / 835(0.4)$ \\
\hline \multicolumn{7}{|l|}{ ECG values } \\
\hline \multicolumn{7}{|l|}{ RR interval (ms) } \\
\hline$<500$ and decreased $\geq 200$ & $0 / 541$ & $0 / 367$ & $0 / 253$ & $0 / 111$ & $0 / 188$ & $0 / 919$ \\
\hline$>1200$ and increased $\geq 200$ & $7 / 541(1.3)$ & $11 / 367(3.0)$ & $3 / 253(1.2)$ & $3 / 111(2.7)$ & $3 / 188(1.6)$ & $20 / 919(2.2)$ \\
\hline \multicolumn{7}{|l|}{ PR interval (ms) } \\
\hline$<120$ & $6 / 539(1.1)$ & $3 / 367(0.8)$ & $8 / 252$ (3.2) & $6 / 111(5.4)$ & 4/187 (2.1) & 21/917 (2.3) \\
\hline$\geq 250$ & $3 / 539(0.6)$ & $0 / 367$ & $2 / 252(0.8)$ & $1 / 111(0.9)$ & $0 / 187$ & $3 / 917(0.3)$ \\
\hline \multicolumn{7}{|l|}{ QRS interval (ms) } \\
\hline$<40$ & $0 / 542$ & $0 / 367$ & $0 / 253$ & $0 / 111$ & $0 / 187$ & $0 / 918$ \\
\hline$>150$ & $4 / 542(0.7)$ & $2 / 367(0.5)$ & $0 / 253$ & $0 / 111$ & $1 / 187(0.5)$ & $3 / 918(0.3)$ \\
\hline \multicolumn{7}{|l|}{ QT } \\
\hline$>450 \mathrm{~ms}$ & $42 / 540(7.8)$ & $36 / 367(9.8)$ & $13 / 253(5.1)$ & $8 / 110(7.3)$ & $11 / 187(5.9)$ & 68/917 (7.4) \\
\hline$>480 \mathrm{~ms}$ & $11 / 540(2.0)$ & $2 / 367(0.5)$ & $0 / 253$ & $1 / 110(0.9)$ & $1 / 187(0.5)$ & $4 / 917(0.4)$ \\
\hline$>500 \mathrm{~ms}$ & $2 / 540(0.4)$ & $2 / 367(0.5)$ & $0 / 253$ & $0 / 110$ & $0 / 187$ & $2 / 917(0.2)$ \\
\hline Increase from baseline $>30 \mathrm{~ms}$ & $65 / 535(12.1)$ & $45 / 366(12.3)$ & $38 / 253(15.0)$ & $15 / 109(13.8)$ & 25/186 (13.4) & $123 / 914(13.5)$ \\
\hline Increase from baseline $>60 \mathrm{~ms}$ & $8 / 535(1.5)$ & $3 / 366(0.8)$ & $2 / 253(0.8)$ & $1 / 109(0.9)$ & $0 / 186$ & 6/914 (0.7) \\
\hline \multicolumn{7}{|l|}{ QTCB } \\
\hline$>450 \mathrm{~ms}$ & $67 / 540(12.4)$ & $47 / 367(12.8)$ & 25/253 (9.9) & $12 / 110(10.9)$ & $15 / 187(8.0)$ & 99/917 (10.8) \\
\hline$>480 \mathrm{~ms}$ & $5 / 540(0.9)$ & $5 / 367(1.4)$ & $0 / 253$ & $1 / 110(0.9)$ & $0 / 187$ & 6/917 (0.7) \\
\hline$>500 \mathrm{~ms}$ & $1 / 540(0.2)$ & $2 / 367(0.5)$ & $0 / 253$ & $1 / 110(0.9)$ & $0 / 187$ & $3 / 917(0.3)$ \\
\hline Increase from baseline $>30 \mathrm{~ms}$ & $33 / 535(6.2)$ & 28/366 (7.7) & $11 / 253(4.3)$ & $7 / 109(6.4)$ & $9 / 186(4.8)$ & $55 / 914(6.0)$ \\
\hline Increase from baseline $>60 \mathrm{~ms}$ & 2/535 (0.4) & $1 / 366(0.3)$ & $2 / 253(0.8)$ & $0 / 109$ & $0 / 186$ & $3 / 914(0.3)$ \\
\hline \multicolumn{7}{|l|}{ QTcF } \\
\hline$>450 \mathrm{~ms}$ & $30 / 540(5.6)$ & $25 / 367(6.8)$ & $10 / 253(4.0)$ & $4 / 110(3.6)$ & $4 / 187(2.1)$ & $43 / 917(4.7)$ \\
\hline$>480 \mathrm{~ms}$ & $3 / 540(0.6)$ & $2 / 367(0.5)$ & $0 / 253$ & $0 / 110$ & $0 / 187$ & $2 / 917(0.2)$ \\
\hline$>500 \mathrm{~ms}$ & $0 / 540$ & $0 / 367$ & $0 / 253$ & $0 / 110$ & $0 / 187$ & 0/917 \\
\hline Increase from baseline $>30 \mathrm{~ms}$ & $17 / 535(3.2)$ & $11 / 366(3.0)$ & $10 / 253(4.0)$ & $4 / 109(3.7)$ & $5 / 186(2.7)$ & $30 / 914(3.3)$ \\
\hline Increase from baseline $>60 \mathrm{~ms}$ & $0 / 535$ & $1 / 366(0.3)$ & $0 / 253$ & $0 / 109$ & $0 / 186$ & $1 / 914(0.1)$ \\
\hline Liver enzymes & & & & & & \\
\hline Total bilirubin ( $\mu \mathrm{mol} / \mathrm{L})$ & & & & & & \\
\hline$\geq 34.2$ & $0 / 547$ & $2 / 368(0.5)$ & $1 / 252(0.4)$ & $0 / 110$ & $0 / 189$ & 3/919 (0.3) \\
\hline
\end{tabular}


TABLE 7. Continued

\begin{tabular}{|c|c|c|c|c|c|c|}
\hline Patients, n/N (\%) & $\begin{array}{l}\text { Placebo } \\
(\mathrm{N}=561)\end{array}$ & $\begin{array}{l}\text { Vortioxetine } 5 \mathrm{mg} \\
\quad(n=376)\end{array}$ & $\begin{array}{l}\text { Vortioxetine } 10 \mathrm{mg} \\
\quad(\mathrm{n}=259)\end{array}$ & $\begin{array}{l}\text { Vortioxetine } 15 \mathrm{mg} \\
\quad(\mathrm{n}=118)\end{array}$ & $\begin{array}{l}\text { Vortioxetine } 20 \mathrm{mg} \\
\quad(\mathrm{n}=194)\end{array}$ & $\begin{array}{l}\text { Vortioxetine all doses } \\
\qquad(\mathrm{N}=947)\end{array}$ \\
\hline \multicolumn{7}{|l|}{ Alkaline phosphatase (U/L) } \\
\hline$\geq 3 \times U L N$ & $0 / 547$ & $0 / 368$ & $0 / 252$ & $0 / 110$ & $0 / 189$ & $0 / 919$ \\
\hline \multicolumn{7}{|l|}{ Aspartate aminotransferase (U/L) } \\
\hline$\geq 3 \times$ ULN & $1 / 546(0.2)$ & $3 / 368(0.8)$ & $0 / 252$ & $0 / 110$ & $0 / 189$ & $3 / 919(0.3)$ \\
\hline \multicolumn{7}{|l|}{ Alanine aminotransferase (U/L) } \\
\hline$\geq 3 \times$ ULN & $1 / 546(0.2)$ & $1 / 368(0.3)$ & $0 / 252$ & $0 / 110$ & $0 / 189$ & $1 / 919(0.1)$ \\
\hline \multicolumn{7}{|c|}{ Gamma-glutamyl transpeptidase (U/L) } \\
\hline$\geq 3 \times$ ULN & $8 / 406(2.0)$ & $5 / 223(2.2)$ & 6/188 (3.2) & $1 / 110(0.9)$ & 2/189 (1.1) & $14 / 710(2.0)$ \\
\hline \multicolumn{7}{|l|}{ Body weight } \\
\hline \multicolumn{7}{|l|}{ Weight (kg) } \\
\hline Mean change (SD) * & $0.05(1.909)$ & $-0.15(1.843)$ & $0.02(1.712)$ & $-0.42(2.040)$ & $0.21(1.837)$ & $-0.06(1.839)$ \\
\hline Decrease from baseline $\geq 7 \%$ & $4 / 546(0.7)$ & $1 / 368(0.3)$ & $1 / 253(0.4)$ & 1/111 (0.9) & $0 / 189$ & $3 / 921(0.3)$ \\
\hline Increase from baseline $\geq 7 \%$ & $5 / 546(0.9)$ & $4 / 368(1.1)$ & $1 / 253(0.4)$ & $0 / 111$ & 2/189 (1.1) & $7 / 921(0.8)$ \\
\hline
\end{tabular}

* Mean change from baseline to final visit (standard deviation). Values measured more than 7 days after the last dose of double-blind study medication are not included in the analysis. Summary based on number (\%) of patients with $\geq 1$ potentially clinically significant value during the treatment period. Standing and supine measurements were collected for all studies except NCT01255787, where only sitting measurements were collected (study omitted). BP - blood pressure; bpm - beats per minute; ECG - electrocardiogram; $\mathrm{mmHg}$ - millimeters of mercury; SD - standard deviation; ULN - upper limit of normal.

\section{Conclusions}

Results from this meta-analysis provide evidence that vortioxetine $5-20 \mathrm{mg} /$ day is efficacious and well tolerated in patients with MDD aged 55 years and older. The meta-analysis of clinical efficacy suggests a doseresponse relationship with vortioxetine $5 \mathrm{mg}, 10 \mathrm{mg}$, and $20 \mathrm{mg}$, as demonstrated by the difference versus placebo in change from baseline on MADRS total score. In this MDD patient population with a variety of comorbid medical conditions and taking a variety of concomitant medications, the safety assessment suggests that vortioxetine is well tolerated.

\section{Disclosures}

At the time of study, George Nomikos and Dapo Tomori were employees of Takeda Development Center Americas. Wei Zhong, John Affinito and William Palo are employees of Takeda Development Center Americas.

\section{REFERENCES:}

1. United Nations Department of Economic and Social Affairs, Population Division. World Population Prospects: The 2015 Revision. Custom data acquired via website http://esa.un.org/ unpd/wpp/DataQuery/. Accessed February 2, 2016.

2. WHO Mental Health in the Elderly. 2015. http://www.who.int/ mediacentre/factsheets/fs381/en/. Accessed November 9, 2015.

3. United States Census Bureau. 2012 National Population Projections. 2012. https://www.census.gov/population/projections/data/ national/2012.html. Accessed November 24, 2015.
4. Colby S, Ortman J. Projections of the size and composition of the U.S. population: 2014 to 2060 , current population reports, P25-1143, U.S. Census Bureau, Washington, DC; 2014.

5. Reynolds K, Pietrzak RH, El-Gabalawy R, Mackenzie CS, Sareen J. Prevalence of psychiatric disorders in U.S. older adults: findings from a nationally representative survey. World Psychiatry. 2015; 14(1): 74-81.

6. Byers AL, Yaffe K, Covinsky KE, Friedman MB, Bruce ML. High occurrence of mood and anxiety disorders among older adults: the National Comorbidity Survey Replication. Arch Gen Psychiatry. 2010; 67(5): 489-496.

7. Lin JH, Huang MW, Wang DW, et al. Late-life depression and quality of life in a geriatric evaluation and management unit: an exploratory study. BMC Geriatr. 2014; 14 : 77.

8. Zivin K, Wharton T, Rostant O. The economic, public health, and caregiver burden of late-life depression. Psychiatr Clin North Am. 2013; 36(4): 631-649.

9. Bock JO, Luppa M, Brettschneider C, et al. Impact of depression on health care utilization and costs among multimorbid patients-from the MultiCare Cohort Study. PLoS One. 2014; 9(3): e91973.

10. Laborde-Lahoz P, El-Gabalawy R, Kinley J, et al. Subsyndromal depression among older adults in the USA: prevalence, comorbidity, and risk for new-onset psychiatric disorders in late life. Int J Geriatr Psychiatry. 2015; 30(7): 677-685.

11. Steffens DC, Fisher GG, Langa KM, Potter GG, Plassman BL. Prevalence of depression among older Americans: the Aging, Demographics and Memory Study. Int Psychogeriatr. 2009; 21(5): 879-888.

12. Reinlieb M, Ercoli LM, Siddarth P, St Cyr N, Lavretsky H. The patterns of cognitive and functional impairment in amnestic and non-amnestic mild cognitive impairment in geriatric depression. Am J Geriatr Psychiatry. 2014; 22(12): 1487-1495.

13. Bhalla RK, Butters MA, Becker JT, et al. Patterns of mild cognitive impairment after treatment of depression in the elderly. Am J Geriatr Psychiatry. 2009; 17(4): 308-316.

14. Callahan CM, Wolinsky FD, Stump TE, et al. Mortality, symptoms, and functional impairment in late-life depression. J Gen Intern Med. 1998; 13(11): 746-752. 
15. Hybels CF, Pieper CF, Payne ME, Steffens DC. Late-life depression modifies the association between cerebral white matter hyperintensities and functional decline among older adults. Am J Geriatr Psychiatry. 2016; 24(1): 42-49.

16. Sinnige J, Korevaar JC, Westert GP, et al. Multimorbidity patterns in a primary care population aged 55 years and over. Fam Pract. 2015; 32(5): 505-513.

17. Gunn JM, Ayton DR, Densley K, et al. The association between chronic illness, multimorbidity and depressive symptoms in an Australian primary care cohort. Soc Psychiatry Psychiatr Epidemiol. 2012; 47(2): 175-184.

18. Ryu E, Chamberlain AM, Pendegraft RS, et al. Quantifying the impact of chronic conditions on a diagnosis of major depressive disorder in adults: a cohort study using linked electronic medical records. BMC Psychiatry. 2016; 16(1): 1-9.

19. Gallo JJ, Bogner HR, Morales KH, et al. Depression, cardiovascular disease, diabetes, and two-year mortality among older, primary-care patients. Am J Geriatr Psychiatry. 2005; 13(9): 748-755.

20. Calati R, Salvina Signorelli M, Balestri M, et al. Antidepressants in elderly: metaregression of double-blind, randomized clinical trials. J Affect Disord. 2013; 147(1-3): 1-8.

21. Nelson JC, Delucchi K, Schneider LS. Anxiety does not predict response to antidepressant treatment in late life depression: results of a meta-analysis. Int J Geriatr Psychiatry. 2009; 24(5): 539-544.

22. Pae CU, Wang SM, Han C, et al. Vortioxetine, a multimodal antidepressant for generalized anxiety disorder: a systematic review and meta-analysis. J Psychiatr Res. 2015; 64: 88-98.

23. Sultana J, Spina E, Trifirò G. Antidepressant use in the elderly: the role of pharmacodynamics and pharmacokinetics in drug safety. Expert Opin Drug Metab Toxicol. 2015; 11(6): 883-892.

24. Beach SR, Kostis WJ, Celano CM, et al. Meta-analysis of selective serotonin reuptake inhibitor-associated QTc prolongation. J Clin Psychiatry. 2014; 75(5): e441-e449.

25. FDA Drug Safety Communication. Revised recommendations for Celexa (citalopram hydrobromide) related to a potential risk of abnormal heart rhythms with high doses. 2012. http://www.fda.gov/ Drugs/DrugSafety/ucm297391.htm. Accessed November 1, 2015.

26. Moura C, Bernatsky S, Abrahamowicz M, et al. Antidepressant use and 10-year incident fracture risk: the population-based Canadian Multicentre Osteoporosis Study (CaMoS). Osteoporos Int. 2014; 25(5): 1473-1481.

27. Giorlando F, Teister J, Dodd S, Udina M, Berk M. Hyponatraemia: an audit of aged psychiatry patients taking SSRIs and SNRIs. Curr Drug Saf. $2013 ; \mathbf{8}(3)$ : 175-180.

28. Tedeschini E, Levkovitz Y, Iovieno N, et al. Efficacy of antidepressants for late-life depression: a meta-analysis and meta-regression of placebo-controlled randomized trials. J Clin Psychiatry. 2011; 72(12): 1660-1668.

29. Nelson JC, Delucchi K, Schneider LS. Efficacy of second generation antidepressants in late-life depression: a meta-analysis of the evidence. Am J Geriatr Psychiatry. 2008; 16(7): 558-567.

30. Katona C, Hansen T, Olsen CK. A randomized, double-blind, placebo-controlled, duloxetine-referenced, fixed-dose study comparing the efficacy and safety of Lu AA21004 in elderly patients with major depressive disorder. Int Clin Psychopharmacol. 2012; 27(4): 215-223.

31. Bang-Andersen B, Ruhland T, Jorgensen M, et al. Discovery of 1-[2-(2,4-dimethylphenylsulfanyl)phenyl]piperazine (Lu AA21004): a novel multimodal compound for the treatment of major depressive disorder. J Med Chem. 2011; 54(9): 3206-3221.

32. Mork A, Pehrson A, Brennum LT, et al. Pharmacological effects of Lu AA21004: a novel multimodal compound for the treatment of major depressive disorder. J Pharmacol Exp Ther. 2012; 340(3): 666-675.
33. Westrich $\mathrm{L}$, Pehrson $\mathrm{A}$, Zhong $\mathrm{H}$, et al. In vitro and in vivo effects for the multimodal antidepressant vortioxetine (Lu AA21004) at human and rat targets. Int J Psychiatry Clin Pract. 2012; 16(S1): 47.

34. Alvarez E, Perez V, Dragheim M, Loft H, Artigas F. A double-blind, randomized, placebo-controlled, active reference study of $\mathrm{Lu}$ AA21004 in patients with major depressive disorder. Int J Neuropsychopharmacol. 2012; 15(5): 589-600.

35. Baldwin DS, Loft H, Dragheim M. A randomised, double-blind, placebo controlled, duloxetine-referenced, fixed-dose study of three dosages of Lu AA21004 in acute treatment of major depressive disorder (MDD). Eur Neuropsychopharmacol. 2012; 22(7): $482-491$.

36. Boulenger JP, Loft H, Olsen CK. Efficacy and safety of vortioxetine (Lu AA21004), 15 and 20mg/day: a randomized, double-blind, placebo-controlled, duloxetine-referenced study in the acute treatment of adult patients with major depressive disorder. Int Clin Psychopharmacol. 2014; 29(3): 138-149.

37. Henigsberg N, Mahableshwarkar AR, Jacobsen P, Chen Y, Thase ME. A randomized, double-blind, placebo-controlled 8-week trial of the efficacy and tolerability of multiple doses of Lu AA21004 in adults with major depressive disorder. J Clin Psychiatry. 2012; 73(7): 953-959.

38. Jacobsen PL, Mahableshwarkar AR, Serenko M, Chan S, Trivedi M. A randomized, double-blind, placebo-controlled study of the efficacy and safety of vortioxetine $10 \mathrm{mg}$ and $20 \mathrm{mg}$ in adults with major depressive disorder. J Clin Psychiatry. 2015; 76(5): 575-582.

39. Jain R, Mahableshwarkar AR, Jacobsen P, Chen Y, Thase ME. A randomized, double-blind, placebo-controlled 6-wk trial of the efficacy and tolerability of $5 \mathrm{mg}$ vortioxetine in adults with major depressive disorder. Int J Neuropsychopharmacol. 2013; 16(2): 313-321.

40. Mahableshwarkar AR, Jacobsen PL, Chen Y, Serenko M, Trivedi M. A randomized, double-blind, duloxetine-referenced study comparing efficacy and tolerability of 2 fixed doses of vortioxetine in the acute treatment of adults with MDD. Psychopharmacology (Berl). 2015; 232(11): 2061-2070.

41. Mahableshwarkar AR, Jacobsen PL, Chen Y. A randomized, doubleblind trial of $2.5 \mathrm{mg}$ and $5 \mathrm{mg}$ vortioxetine ( $\mathrm{Lu} \mathrm{AA21004)} \mathrm{versus}$ placebo for 8 weeks in adults with major depressive disorder. Curr Med Res Opin. 2013; 29(3): 217-226.

42. McIntyre RS, Lophaven S, Olsen CK. A randomized, double-blind, placebo-controlled study of vortioxetine on cognitive function in depressed adults. Int J Neuropsychopharmacol. 2014; 17(10): 1557-1567.

43. Takeda. A multinational, randomized, double-blind, placebocontrolled, dose ranging study to assess the efficacy and safety of $\mathrm{Lu}$ AA21004 in patients with major depressive disorder. 2013. NCT01255787. Available at: https://clinicaltrials.gov/ct2/show/ NCT01255787. Accessed February 23, 2016

44. Mahableshwarkar AR, Jacobsen P, Serenko M, Chen Y, Trivedi M. A randomized, double-blind, placebo-controlled study of the efficacy and safety of 2 doses of vortioxetine in adults with major depressive disorder. J Clin Psychiatry. 2015; 76(5): 583-591.

45. World Medical Association (WMA). Declaration of Helsinki: ethica principles for medical research involving human subjects. World Medical Association (WMA). 2008. http://www.wma.net/en/ 30publications/10policies/b3/17c.pdf. Accessed May 10, 2016

46. ICH Harmonised Tripartite Guideline E6(R1): Guideline for Good Clinical Practice. 1996. http://www.hadassah.org.il/media/ 1875250/e6_r1__guideline.pdf.

47. Montgomery SA, Asberg M. A new depression scale designed to be sensitive to change. Br J Psychiatry. 1979; 134(4): 382-389.

48. Guy W. Clinical Global Impressions (028-CGI). In: Guy W, ed. ECDEU Assessment Manual for Psychopharmacology. Vol Revised. 
Rockville, MD: U.S. Dept. of Health, Education, and Welfare, Public Health Service, Alcohol, Drug Abuse, and Mental Health Administration; 1976.

49. Hamilton M. Development of a rating scale for primary depressive illness. Br J Soc Clin Psychol. 1967; 6(4): 278-296.

50. Wechsler D. Wechsler Adult Intelligence Scale ${ }^{\circledR}$. Third Edition (WAIS ${ }^{\circledR}$-III). San Antonio, TX: Psychologial Corporation; 1997. http://www.pearsonclinical.com/psychology/products/ 100000243/wechsler-adult-intelligence-scale-third-edition-wais-iii. html. Accessed July 28, 2015.

51. Lezak MD, Howieson DB, Loring DW. Neuropsychological Assessment. 4th ed., Vol 4. New York: Oxford University Press; 2004.

52. Anderson TM, Slade T, Andrews G, Sachdev PS. DSM-IV major depressive episode in the elderly: the relationship between the number and the type of depressive symptoms and impairment. $J$ Affect Disord. 2009; 117(1-2): 55-62.

53. Pae CU, Wang SM, Han C, et al. Vortioxetine: a meta-analysis of 12 short-term, randomized, placebo-controlled clinical trials for the treatment of major depressive disorder. J Psychiatry Neurosci. 2015; 40(3): 174-186.
54. Meeker AS, Herink MC, Haxby DG, Hartung DM. The safety and efficacy of vortioxetine for acute treatment of major depressive disorder: a systematic review and meta-analysis. Syst Rev. 2015; 4: 21 .

55. Berhan A, Barker A. Vortioxetine in the treatment of adult patients with major depressive disorder: a meta-analysis of randomized double-blind controlled trials. BMC Psychiatry. 2014; 14(1): 276.

56. Vieta E, Pappadopulos E, Mandel FS, Lombardo I. Impact of geographical and cultural factors on clinical trials in acute mania: lessons from a ziprasidone and haloperidol placebocontrolled study. Int J Neuropsychopharmacol. 2011; 14(8): 1017-1027.

57. Khin NA, Chen YF, Yang Y, Yang P, Laughren TP. Exploratory analyses of efficacy data from major depressive disorder trials submitted to the US Food and Drug Administration in support of new drug applications. J Clin Psychiatry. 2011; 72(4): 464-472.

58. Vazquez G, Baldessarini RJ, Yildiz A, et al. Multisite international collaborative clinical trials in mania. Int J Neuropsychopharmacol. 2011; 14(8): 1013-1016.

59. Hardy RJ, Thompson SG. Detecting and describing heterogeneity in meta-analysis. Stat Med. 1998; 17(8): 841-856. 